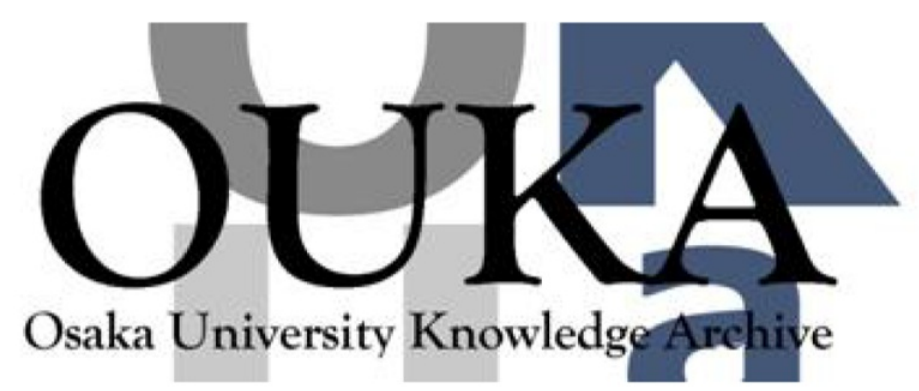

\begin{tabular}{|c|l|}
\hline Title & $\begin{array}{l}\text { Analysis of a kinematic model for ion transport } \\
\text { in rf plasma sheaths }\end{array}$ \\
\hline Author(s) & $\begin{array}{l}\text { Farouki, Rida T.; Hamaguchi, Satoshi; Dalvie, } \\
\text { Manoj }\end{array}$ \\
\hline Citation & Physical Review A. 45(8) p. 5913-p.5928 \\
\hline Issue Date & $1992-04$ \\
\hline oaire:version & VoR \\
\hline URL & https://hdl. handle. net/11094/78517 \\
\hline rights & $\begin{array}{l}\text { Copyright (1992) by the American Physical } \\
\text { Society. }\end{array}$ \\
\hline Note & \\
\hline
\end{tabular}

Osaka University Knowledge Archive : OUKA

https://ir. Library. osaka-u. ac. jp/

Osaka University 


\title{
Analysis of a kinematic model for ion transport in rf plasma sheaths
}

\author{
Rida T. Farouki, Satoshi Hamaguchi, and Manoj Dalvie \\ IBM Thomas J. Watson Research Center, P.O. Box 218, Yorktown Heights, New York 10598
}

(Received 12 September 1991)

\begin{abstract}
An idealized model for ion transport across an oscillating plasma sheath is analyzed to obtain insight into qualitative features of the ion energy distributions observed in low-pressure rf discharges. The sheath is characterized by a constant electric field over an extent that varies sinusoidally with time, and ions incident on it correspond to a monoenergetic flux independent of phase $\phi$ in the rf cycle. The dimensionless parameters $\alpha=q V_{s} / m \omega^{2} d^{2}$ and $\beta=v_{0} / \omega d$ (where $d$ and $V_{s}$ are the mean sheath thickness and potential drop, $\omega$ is the excitation frequency, and $v_{0}$ and $q / m$ are the incoming ion speed and charge-to-mass ratio) govern the ion trajectories, which are found to divide into groups, delimited by two "critical" values of $\phi$, that undergo $N$ and $N+1$ encounters with the field. The first critical phase depends only weakly on $\beta$, whereas the second is sensitive to both $\alpha$ and $\beta$ and cycles continuously as these parameters diminish. Correspondingly, within the "transition regime" where $\alpha$ and $\beta$ are neither very small nor greater than (or comparable to) unity, the precise form of the incident-ion energy spectrum exhibits rapid variations, superposed on a systematic narrowing, as the frequency $\omega$ is increased.
\end{abstract}

PACS number(s): 52.25.Fi, 52.65. $+\mathrm{z}$, 52.80.Pi

\section{INTRODUCTION}

A striking feature of the incident-ion energy distributions measured at the electrodes of low-pressure radiofrequency (rf) discharges is the "saddle" or "doublepeak" configuration, observed within a range of the excitation frequency that depends on the molecular weight of the ions [1-3]. Typically, the energies of ions of a given species exhibit a rather broad distribution at low frequencies and are concentrated within a narrow "spike" centered on the dc bias at high frequencies; the double-peak structure is characteristic of the transition between these limiting regimes. An understanding of and control over the precise form of the ion energy distributions is of practical interest in the plasma processing of semiconductor materials [4].

The measured energies of the ions reflect their acceleration by the strong electric field of the oscillating plasma sheath. The problem of obtaining a quantitative description of the incident-ion energy distribution is intimately coupled to that of understanding the timedependent sheath structure, and is especially difficult to treat in a self-consistent manner (even in the collisionless case) at frequencies within the transition regime. Nevertheless, a number of theoretical studies based on the numerical integration of ion trajectories across sheaths with an assumed more-or-less "realistic" functional form for the time-dependent electric field have succeeded in reproducing the salient features of observed ion energy distributions.

Such studies include analysis of (numerically determined) relationships between the final energies of ions and the "phase" of the sheath oscillation when they are introduced [5-7], assuming one-dimensional collisionless ion motion with a monoenergetic incoming flux that is independent of time in the rf cycle, and Monte Carlo simulations [8-11], which allow the effects of collisions [12] and/or a distribution for the initial energies of ions to be incorporated. A sheath field that is linearly increasing or of Child-Langmuir form is generally assumed in these models, the sheath thickness being taken as constant, sinusoidally varying, or having a temporal variation chosen to match a model waveform for the potential drop across the sheath $[7,13]$. Ion energy distributions have also been measured in self-consistent, particle-in-cell simulations of $\mathrm{rf}$ discharges $[14,15]$.

The qualitative agreement of the results of such models with experimental data under a variety of assumptions regarding the sheath dynamics suggests that, in the collisionless regime, a simple universal mechanism governs the variation of the overall shape of the energy distributions with frequency. Thus, in view of the current primitive state of quantitative models for the self-consistent structure of $\mathrm{rf}$ sheaths at arbitrary excitation frequencies [16], it seems desirable to reexamine the rf-sheath iontransport problem in the context of some rather severe simplifications that may serve to elucidate such a mechanism.

To the extent that they are credible, detailed profiles for the spatial and temporal variation of the sheath field can improve quantitative comparisons with measured distributions. In terms of gaining basic insight into the origin of the observed forms of the energy distributions, however, they become a hindrance since they greatly complicate the analysis. These considerations have motivated us to explore in detail an idealized model of the rf-sheath ion-transport problem, as described below.

The analysis of this model reveals some interesting physical effects, and offers an intuitive explanation for the observed qualitative shapes of the ion energy distributions. (It may also yield useful insight for the formulation of more sophisticated models, in terms of corroborating the validity of certain assumptions regarding ion motion in an oscillating sheath.) Our main results are summarized in Sec. V; the reader that has no patience for details may proceed directly there after working through the basic model description that follows. 


\section{THE ION-TRANSPORT MODEL}

The following physical parameters characterize our idealized model: $v_{0}$ is the initial ion speed, $d$ is the mean sheath thickness, $V_{s}$ is the mean potential drop across the sheath, $\omega$ is the angular frequency of the rf excitation, and $q / m$ is the ion charge-to-mass ratio. The extent of the sheath is $-d \leq z \leq+d$, ions entering from the plasma with speed $v_{0}$ at $z=-d$ and impinging on the cathode at $z=+d$ (typically, $v_{0}$ is identified with the Bohm velocity $\sqrt{k_{B} T_{e} / m}$, where $k_{B}$ is the Boltzmann constant and $T_{e}$ is the electron temperature for the plasma bulk [17]). The motion of the plasma-sheath boundary is described by

$$
z_{s}(t)=-d \cos (\omega t+\phi),
$$

where $\phi$ represents the phase of the sheath oscillation at the time $t=0$ that an ion is introduced. We take $\phi$ in the range $-\pi<\phi \leq+\pi$, noting that $z_{s}(0)=-d$ and $\dot{z}_{s}(0)=0$ when $\phi=0$. Otherwise, $z_{s}(0)>-d$ and $\dot{z}_{s}(0)$ is negative or positive according to whether $\phi$ is negative or positive.

For the time-dependent sheath field, we assume the simple model

$$
E(z, t)= \begin{cases}E_{s} & \text { if } z \geq z_{s}(t) \\ 0 & \text { otherwise }\end{cases}
$$

where $E_{s}$ represents the mean field $V_{s} / d$, and the location $z_{s}(t)$ of the plasma sheath boundary is given by (1). Thus, the ion equation of motion may be written as

$$
\frac{d^{2} z}{d t^{2}}=\frac{q E_{s}}{m} H(z+d \cos (\omega t+\phi)),
$$

where $H$ denotes the Heaviside step function

$$
H(x)= \begin{cases}1 & \text { if } x \geq 0 \\ 0 & \text { otherwise } .\end{cases}
$$

Writing $v=d z / d t$, a complete solution to the iontransport problem is given by integrating (3), for each $\phi$, subject to the initial conditions

$$
z(0)=-d \text { and } v(0)=v_{0} .
$$

The difficulty in integrating (3) stems from its nonautonomous nature, i.e., the explicit dependence of the righthand-side "forcing" term on the time $t$ as well as the spatial coordinate $z$. The oscillating sheath model described by Eqs. (1) and (2) has been used by Goedde, Lichtenberg, and Lieberman [18], but in the context of studying the stochastic heating of electrons due to reflections between two sheaths rather than ion transport through a sheath.

A simpler model, in which $z_{s}$ is held fixed and $E_{s}$ oscillates sinusoidally with time, can be integrated explicitly [19] but is less appropriate to the present context. The high mobility of the electron gas enables it to respond almost instantaneously to the rf excitation, giving rise to a "pumping" action in which electrons are alternatively attracted toward and repelled from the cathode. Associated with this phenomenon, the presence of a relatively sharp oscillating plasma-sheath boundary is expected to be a key feature of realistic ion transport models.

\section{A. Dimensionless parameters}

It will be convenient to introduce the dimensionless variables

$$
\tau=\omega t, \quad \xi=\frac{z}{d}, \quad \eta=\frac{v}{\omega d},
$$

in terms of which equations (3) and (5) become

$$
\begin{aligned}
& \frac{d^{2} \xi}{d \tau^{2}}=\alpha H[\xi+\cos (\tau+\phi)], \\
& \xi(0)=-1 \text { and } \eta(0)=\beta .
\end{aligned}
$$

The dimensionless constants

$$
\alpha=\frac{q V_{s}}{m \omega^{2} d^{2}} \text { and } \beta=\frac{v_{0}}{\omega d}
$$

that arise in (7) and (8) can be regarded as measures of the ion acceleration due to the sheath field, relative to that of the plasma-sheath boundary (at its greatest excursion), and of the incoming ion speed relative to that of the plasma-sheath boundary (at its mean position), respectively.

We call $\alpha$ the acceleration parameter and $\beta$ the initial velocity parameter. In our model, these parameters uniquely characterize the solution to the ion-transport problem, and the resulting energy distribution of the ion flux at the cathode. Since they are defined in terms of simple characteristics of the sheath, it should be easy to compute "equivalent" values of these parameters for more sophisticated sheath models, and hence to compare the results of our analysis with the energy distributions generated by more "realistic" profiles for the spatial and temporal variation of the sheath field.

We also write $\mathscr{E}=E / E_{s}$ and $\Phi=V / V_{s}$ for the dimensionless electric field and potential, and $\xi_{s}=z_{s} / d$ for the location of the plasma-sheath boundary. Ion kinetic energies are measured relative to the maximum possible energy of an ion arriving at the cathode,

$$
\varepsilon=\frac{\frac{1}{2} m v^{2}}{2 q V_{s}+\frac{1}{2} m v_{0}^{2}}=\frac{\eta^{2}}{4 \alpha+\beta^{2}} .
$$

Thus, the dimensionless energies of ions that impinge on the cathode are in the range

$$
\varepsilon_{\min }=\frac{\beta^{2}}{4 \alpha+\beta^{2}} \leq \varepsilon \leq 1=\varepsilon_{\max } .
$$

Although $\varepsilon_{\max }$ is unity by definition, we nevertheless write it symbolically at times to emphasize certain symmetries.

Another dimensionless parameter that is frequently used to characterize the rf-sheath ion-transport problem is the ratio

$$
\Omega=\frac{\left\langle T_{\omega}(\phi)\right\rangle}{2 \pi / \omega}
$$

of the "mean ion-transit time" to the period of the rf cycle [where $T_{\omega}(\phi)$ denotes the time at which the solution $z(t)$ to (3) and (5) reaches $+d$, and $\left\langle T_{\omega}(\phi)\right\rangle$ is the average of these times over all $\phi]$. In general, $\Omega$ has no simple 
expression in terms of the dimensionless parameters $\alpha$ and $\beta$. However, taking $\left(2 q V_{s} / m+v_{0}^{2}\right)^{1 / 2}$ as a "typical" ion velocity gives the crude estimate

$$
\Omega(\alpha, \beta) \approx \frac{1}{\pi\left(2 \alpha+\beta^{2}\right)^{1 / 2}} .
$$

In descriptive terms, the solution of (7) subject to (8) for a given phase $\phi$ is straightforward: the motion consists of an alternating sequence of constant-velocity and constant-acceleration intervals, $\xi$ exhibiting a strictly monotone increase from the initial value -1 until the integration is truncated at $\xi=+1$. Although this kinematic behavior is not describable by a single analytic expression, it can nevertheless be determined in a robust, algorithmic manner (see the Appendix).

If it is assumed that ions entering the oscillating sheath are characterized by a constant (time-independent) flux at $\xi=-1$, the phase angle $\phi$ must be regarded as a random variable uniformly distributed between $-\pi$ and $+\pi$. Thus, we are interested here in characterizing the behavior of the family of solutions to (7) generated by varying $\phi$ continuously, and investigating the role of the parameters (9) in influencing this family of solutions.

\section{B. Limiting regimes of the model}

The limiting regimes $\omega \rightarrow 0$ and $\omega \rightarrow \infty$ of our model admit some analytic treatment, and serve as useful references for comparison with the behavior at intermediate frequencies.

\section{The low-frequency limit}

In the low-frequency limit $(\alpha \gg>1$ and $\beta>>1)$ the plasma-sheath boundary is "frozen" at the location $z_{s}=-d \cos \phi$, and the electric field is 0 for $z<z_{s}$ and $E_{s}$ for $z \geq z_{s}$ (all values of the phase $\phi$ between $-\pi$ and $+\pi$ being equally probable). Thus, the ion motion is described by

$$
\xi=-1+\beta \tau \text { and } \eta=\beta
$$

during the "coasting" interval $0 \leq \tau<\tau_{1}$, and by

$\xi=-1+\beta \tau+\frac{\alpha}{2}\left(\tau-\tau_{1}\right)^{2}$ and $\eta=\beta+\alpha\left(\tau-\tau_{1}\right)$

during the acceleration interval $\tau_{1} \leq \tau \leq \tau_{2}$, where $\tau_{1}$ and $\tau_{2}$ are given by

$$
\begin{aligned}
\tau_{1} & =\frac{1-\cos \phi}{\beta}, \\
\tau_{2} & =\tau_{1}+\frac{\left[2 \alpha(1+\cos \phi)+\beta^{2}\right]^{1 / 2}-\beta}{\alpha} .
\end{aligned}
$$

Identifying $\tau_{2}$ with $\omega T_{0}(\phi)$, where $T_{0}(\phi)$ is the lowfrequency transit time for phase $\phi$, we have

$$
\frac{T_{0}(\phi)}{2 \pi / \omega}=\frac{1-\cos \phi}{2 \pi \beta}+\frac{\left[2 \alpha(1+\cos \phi)+\beta^{2}\right]^{1 / 2}-\beta}{2 \pi \alpha} .
$$

Averaging the above over all $\phi$ then gives
$\Omega_{0}(\alpha, \beta)=\frac{1}{2 \pi}\left(\frac{1}{\beta}+\frac{2\left(4 \alpha+\beta^{2}\right)^{1 / 2} E(k)}{\pi \alpha}-\frac{\beta}{\alpha}\right)$

for the limit $\omega \rightarrow 0$ of the ratio (12), where $E$ denotes a complete elliptic integral of the second kind,

$$
\begin{aligned}
& E(k)=\int_{0}^{\pi / 2}\left(1-k^{2} \sin ^{2} \theta\right)^{1 / 2} d \theta \\
& \text { with } k=\left(\frac{4 \alpha}{4 \alpha+\beta^{2}}\right)^{1 / 2} .
\end{aligned}
$$

Note that the value of the integral (19) declines monotonically with $k$ from $E(0)=\pi / 2$ to $E(1)=1$. Expression (18) will be compared to measured values of $\Omega$ at finite $\alpha$ and $\beta$ in Sec. III.

In the low-frequency limit, ions will arrive at all energies between $\varepsilon_{\min }$ and $\varepsilon_{\max }$. They are not uniformly distributed between these extremes, however - they tend to concentrate near $\varepsilon_{\min }$ and $\varepsilon_{\max }$ because, according to (1), the plasma-sheath boundary spends a greater fraction of the rf cycle near $z_{s}=-d$ or $z_{s}=+d$ than near $z_{s}=0$.

A precise form for the time-averaged energy distribution of the incident-ion flux can be derived in the lowfrequency regime, assuming that $\phi$ is random and uniformly distributed between $-\pi$ and $+\pi$. The energy of an ion incident on the cathode at $\xi=+1$ is given by

$$
\varepsilon(\phi)=\frac{2 \alpha(1+\cos \phi)+\beta^{2}}{4 \alpha+\beta^{2}},
$$

and if $d n_{i}$ ions are incident on a unit area of the plane $\xi=-1$ at phases between $\phi$ and $\phi+d \phi$ in a single rf cycle, then an equal number $d n_{i}$ must impinge on a unit area of the cathode at $\xi=+1$ with energies between $\varepsilon$ and $\varepsilon+d \varepsilon$ during that cycle, where $d \varepsilon$ is related to $d \phi$ [20] by (the magnitude of) the derivative of expression (20).

[We assume the solution to the ion-transport problem is periodic-i.e., although the instantaneous ion flux at $\xi=+1$ and the density distribution over $-1 \leq \xi \leq+1$ may vary during a rf cycle, they are identical at a fixed phase $\phi$ within successive cycles. Thus, notwithstanding the fact that the transit time $T$ of an ion varies with the phase $\phi$ on its introduction, over an entire cycle an ion must be received at $\xi=+1$ with energy $\varepsilon(\phi)$ given by (20) to balance each incoming ion at phase $\phi$. Otherwise, a "secular" evolution of the sheath structure will result.]

Introducing the quantities $\varepsilon_{0}=\frac{1}{2}\left(\varepsilon_{\min }+\varepsilon_{\max }\right)$ and $\Delta \varepsilon$ $=\frac{1}{2}\left(\varepsilon_{\max }-\varepsilon_{\text {min }}\right)$ from (11), we can rewrite expression (20) more succinctly as

$$
\varepsilon(\phi)=\varepsilon_{0}+\Delta \varepsilon \cos \phi .
$$

Differentiating (21) and expressing the result in terms of $\varepsilon$ then gives

$$
\frac{d \varepsilon}{d \phi}=-\Delta \varepsilon \sin \phi=\mp\left[1-\left[\frac{\varepsilon-\varepsilon_{0}}{\Delta \varepsilon}\right]^{2}\right]^{1 / 2},
$$

where the choice of sign corresponds to the two phases, $\phi$ and $-\phi$, that yield a given value of $\varepsilon$ in (21).

Thus the energy distribution of the ion flux, averaged 
over a complete rf cycle, is given by

$$
\frac{d n_{i}}{d \varepsilon}=\frac{d n_{i}}{d \phi}\left|\frac{d \varepsilon}{d \phi}\right|_{\phi}^{-1}+\frac{d n_{i}}{d \phi}\left|\frac{d \varepsilon}{d \phi}\right|_{-\phi}^{-1} .
$$

Now on writing $\varepsilon_{0}=\frac{1}{2}\left(\varepsilon_{\min }+\varepsilon_{\max }\right)$ and $\Delta \varepsilon=\frac{1}{2}\left(\varepsilon_{\max }-\varepsilon_{\min }\right)$ in (22) and factoring the argument of the radical, we see that

$$
\left|\frac{d \varepsilon}{d \phi}\right|_{\phi}=\frac{\left[\left(\varepsilon_{\max }-\varepsilon\right)\left(\varepsilon-\varepsilon_{\min }\right)\right]^{1 / 2}}{\Delta \varepsilon}=\left|\frac{d \varepsilon}{d \phi}\right|_{-\phi},
$$

and since (by assumption) $d n_{i} / d \phi$ is just a constant, it is evident that the two terms in (23) make equal contributions, inversely proportional to (24).

Thus, if $\Gamma_{0}(\varepsilon)$ denotes the normalized distribution of the ion flux with respect to energy (averaged over a rf cycle) in the limit $\omega \rightarrow 0$, we have

$$
\Gamma_{0}(\varepsilon) d \varepsilon=\frac{1}{\pi} \frac{d \varepsilon}{\left[\left(\varepsilon_{\max }-\varepsilon\right)\left(\varepsilon-\varepsilon_{\min }\right)\right]^{1 / 2}}
$$

for $\varepsilon_{\min }<\varepsilon<\varepsilon_{\max }$, and $\Gamma_{0}(\varepsilon) d \varepsilon \equiv 0$ otherwise. This distribution exhibits the characteristic double-peak structure -in fact, it is formally singular at $\varepsilon_{\min }$ and $\varepsilon_{\max }$. (These singularities are, of course, "smeared" into spikes of finite height by superposing a finite temperature on the incoming ion beam.) Note also that the energy distribution (25) is symmetric about the median value $\varepsilon_{0}=\frac{1}{2}\left(\varepsilon_{\min }+\varepsilon_{\max }\right)$, i.e., on writing $\varepsilon=(1-\mu) \varepsilon_{\min }+\mu \varepsilon_{\max }$ we observe that $\Gamma_{0}(\mu)=\Gamma_{0}(1-\mu)$ for all $0<\mu<1$.

The analytic form (25) has been compared to distributions obtained from a simulation program, based on the explicit numerical integration of a large number of ion trajectories in arbitrary time-periodic fields, with a random distribution of the initial phase $\phi$ (this was adapted from the Monte Carlo program described in [21]). Figure 1 shows representative results for the parameter values $\alpha=\beta^{2}=1.0,0.5$, and 0.25 (chosen to yield a fixed value $\varepsilon_{\min }=0.2$ for the low-energy cutoff).

In the simulation program, final ion energies are recorded in bins of a finite width $\delta \varepsilon$. Thus, to give a fair comparison of (25) with the simulation results at the lowand high-energy extremes $\varepsilon_{\min }$ and $\varepsilon_{\max }$, we truncate the singularities of $\Gamma_{0}(\varepsilon)$ at finite values $\Gamma_{0, \min }$ and $\Gamma_{0, \max }$ defined such that, over the finite intervals $\left[\varepsilon_{\min }, \varepsilon_{\min }+\delta \varepsilon\right]$ and $\left[\varepsilon_{\max }-\delta \varepsilon, \varepsilon_{\max }\right]$, the area under the analytic form (25) is precisely equal to $\Gamma_{0, \min } \delta \varepsilon$ and $\Gamma_{0 \text {, max }} \delta \varepsilon$, respectively. Thus we have

$$
\begin{aligned}
\Gamma_{0, \max } \delta \varepsilon & =\int_{\varepsilon_{\max }-\delta \varepsilon}^{\varepsilon_{\max }} \Gamma_{0}(\varepsilon) d \varepsilon \\
& =\frac{2}{\pi} \tan ^{-1}\left(\frac{\delta \varepsilon}{\varepsilon_{\max }-\varepsilon_{\min }-\delta \varepsilon}\right)^{1 / 2},
\end{aligned}
$$

and $\Gamma_{0, \min }=\Gamma_{0, \max }$ by the symmetry of (25). We use $\delta \varepsilon=0.01$ in Fig. 1 .

Although the derivation of (25) is based on the assumption that $\alpha \gg 1$ and $\beta \gg 1$, we have found empirically that it yields an excellent description of the energy distribution even at finite values of these parameters. The first

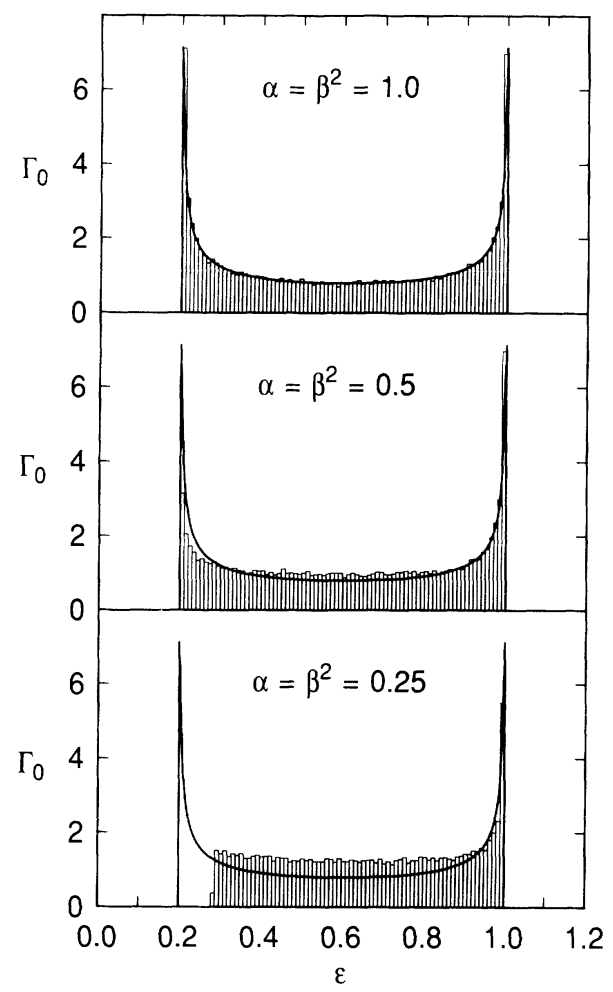

FIG. 1. Comparison of the analytic form (25) for the lowfrequency energy dependence of the ion flux with energy distributions obtained numerically at an increasing sequence of finite frequencies, corresponding to the parameter values $\alpha=\beta^{2}=1.0$, 0.5 , and 0.25 (for which $\varepsilon_{\min }=0.2$ ).

case shown in Fig. 1, for example, shows a remarkably accurate agreement between (25) and the simulations results even when $\alpha=\beta=1$.

In the case $\alpha=\frac{1}{2}$ and $\beta=1 / \sqrt{2}$, however, the true energy distribution is noticeably deficient compared to (25) at $\varepsilon_{\min }$ and has a slight excess at intermediate values, although the high-energy spike is still accurate. With a further reduction of the parameters to $\alpha=\frac{1}{4}$ and $\beta=\frac{1}{2}$, expression (25) fails to offer even a qualitatively correct description of the true energy distribution, which no longer spikes at $\varepsilon_{\min }$. Consistent with the behavior seen in Fig. 1, we will show in Sec. III that the extension of the energy distribution $\Gamma(\varepsilon)$ all the way down to $\varepsilon_{\min }$ is critically dependent on the initial velocity parameter exceeding a special value $\beta_{*} \approx 0.72$ [see Eq. (50)].

A detailed comparison of empirical distributions with the analytic form (25) reveals that the domain of validity of the latter is determined largely by the initial velocity parameter $\beta$. Namely, if $\beta \gtrsim 1$ then $\Gamma_{0}(\varepsilon)$ is in excellent agreement with the true distribution, regardless of the value of $\alpha$. This is perhaps not unexpected, since if $\beta>1$ the incoming ion speed exceeds the maximum speed of the oscillating plasma-sheath boundary-each ion will cross the boundary only once, and changing $\alpha$ cannot alter this fact.

Further, when $\beta<1$, expression (25) still provides an accurate description of the high-energy spike - in the integral sense defined by (26) - until either of the parame- 
ters $\alpha$ or $\beta$ is reduced below about $\frac{1}{2}$.

Finally, note that the energy distribution (25) of the ion flux arises simply from the fact that final ion energy has the dependence $\varepsilon(\phi)=\varepsilon_{0}+\Delta \varepsilon \cos \phi$ on the initial phase angle $\phi$, which is assumed to be uniformly distributed. Thus, in addition to the low-frequency limit of our simple model with a constant field strength and an oscillating sheath thickness, the distribution (25) is also appropriate to other models that give rise to this form for $\varepsilon(\phi)$.

\section{The high-frequency limit}

In the high-frequency limit $(\alpha<<1$ and $\beta<<1)$ the ion motion is determined by the time-averaged field $\overline{\mathscr{E}}(\xi)$, which is simply equal to the fraction of the rf cycle for which $\xi>\xi_{s}(t)$. From (1) and (2) it is readily seen that

$$
\overline{\mathscr{E}}(\xi)=\frac{\pi+2 \sin ^{-1} \xi}{2 \pi}
$$

where we take $\sin ^{-1} \xi \in[-\pi / 2,+\pi / 2]$. Note that $\overline{\mathscr{E}}(-1)=0, \overline{\mathscr{E}}(0)=\frac{1}{2}$, and $\overline{\mathscr{E}}(+1)=1$. Also, the mean value of (27) over the entire extent $-1 \leq \xi \leq+1$ of the sheath is simply $\frac{1}{2}$.

The field (27) may be integrated to give the timeaveraged potential

$$
\bar{\Phi}(\xi)=-\frac{\xi\left(\pi+2 \sin ^{-1} \xi\right)+2\left(1-\xi^{2}\right)^{1 / 2}}{2 \pi},
$$

relative to the reference value $\bar{\Phi}(-1)=0$. From (28) the overall potential drop across the time-averaged sheath is just $\bar{\Phi}(-1)-\bar{\Phi}(+1)=1$ (i.e., $V_{s}$ in dimensional units).

In terms of the potential (28), the dimensionless ion speed $\bar{\eta}(\xi)$ in the mean field may be written as

$$
\bar{\eta}(\xi)=\left[\beta^{2}-2 \alpha \bar{\Phi}(\xi)\right]^{1 / 2} .
$$

Consequently, the quantity (12) may be expressed in the high-frequency limit as the integral

$$
\Omega_{\infty}(\alpha, \beta)=\frac{1}{2 \pi} \int_{-1}^{+1} \frac{1}{\left[\beta^{2}-2 \alpha \bar{\Phi}(\xi)\right]^{1 / 2}} d \xi
$$

provided that $\left(2 \alpha+\beta^{2}\right)^{1 / 2}<<1$. Substituting from (28) and changing the integration variable to $\theta=\sin ^{-1} \xi$ $+\pi / 2$, this integral simplifies to

$\Omega_{\infty}(\alpha, \beta)=\frac{1}{2 \sqrt{2 \pi \alpha}} \int_{0}^{\pi} \frac{\sin \theta}{\left(\sin \theta-\theta \cos \theta+\pi \beta^{2} / 2 \alpha\right)^{1 / 2}} d \theta$.

Expression (31) cannot be resolved into elementary functions of $\alpha$ and $\beta$, although it is easily computed at specific values by a numerical quadrature. We will compare this expression to measured values of $\Omega$ at finite $\alpha$ and $\beta$ in Sec. III.

The ion energy distribution for the high-frequency limit of our model is trivial: all ions arrive with the median energy $\varepsilon_{0}=\frac{1}{2}\left(\varepsilon_{\min }+\varepsilon_{\max }\right)$, and their normalized distribution is simply

$$
\Gamma_{\infty}(\varepsilon) d \varepsilon=\delta\left(\varepsilon-\varepsilon_{0}\right) d \varepsilon .
$$

Elsewhere [22] we have given a more sophisticated treatment of the high-frequency regime, based on an asymptotic expansion of the ion equation of motion in terms of a small parameter equal to the ratio of the rf period to the mean ion-transit time. For large but finite frequencies, the first-order correction to the mean motion yields an energy distribution of finite width. Specifically, in the case of a sheath of constant width and a field strength that is sinusoidally modulated in time, this distribution has the form

$$
\Gamma(\varepsilon) d \varepsilon \propto \frac{d \varepsilon}{\left[\left(\sqrt{\varepsilon_{\max }}-\sqrt{\varepsilon}\right)\left(\sqrt{\varepsilon}-\sqrt{\varepsilon_{\min }}\right)\right]^{1 / 2}}
$$

for $\varepsilon_{\min }<\varepsilon<\varepsilon_{\max }$, where the spread $\varepsilon_{\max }-\varepsilon_{\min }$ and midpoint $\frac{1}{2}\left(\varepsilon_{\min }+\varepsilon_{\max }\right)$ of the distribution are predicted in terms of various sheath characteristics. Although (33) is similar in appearance to (25) for given $\varepsilon_{\min }$ and $\varepsilon_{\max }$, it should be noted that the former is not strictly symmetric and typically is valid only when $\varepsilon_{\max }-\varepsilon_{\min }$ $<<\frac{1}{2}\left(\varepsilon_{\min }+\varepsilon_{\max }\right)$.

The scheme described in [22] is also capable, in principle, of handling our current model (constant field strength, oscillating sheath thickness) but its evaluation for this model is not so straightforward.

\section{ANALYSIS OF THE TRANSPORT PROBLEM}

At intermediate frequencies, i.e., when the magnitude of the parameter

$$
\left(2 \alpha+\beta^{2}\right)^{1 / 2}=\frac{\left(2 q V_{s} / m+v_{0}^{2}\right)^{1 / 2}}{\omega d}
$$

is neither very small nor very large, the family of solutions to (7) and (8) for $-\pi<\phi \leq+\pi$ has a rather complicated nature. Correspondingly, quantities that depend explicitly on the behavior of this family as a whole- such as the ratio $\Omega$ defined by (12) and the incident-ion energy distribution $\Gamma(\varepsilon)$ - have no simple expression in terms of the parameters $\alpha$ and $\beta$.

As noted above, this complication is primarily of a combinatorial nature (in terms of the number of crossings of the plasma-sheath boundary that ions in various ranges of the phase $\phi$ have, etc.).

\section{A. The phase-plane flow}

Ideally, the ion-transport problem would be approached by first solving the Vlasov equation

$$
\frac{\partial f}{\partial \tau}+\eta \frac{\partial f}{\partial \xi}+\alpha H(\xi+\cos \tau) \frac{\partial f}{\partial \eta}=0
$$

(assuming collisionless motion) for the time-dependent distribution function $f(\xi, \eta, \tau)$ in the three-dimensional domain

$-1 \leq \xi \leq+1, \quad \beta \leq \eta \leq\left(4 \alpha+\beta^{2}\right)^{1 / 2}, \quad 0 \leq \tau \leq 2 \pi$,

subject to the boundary condition

$$
f(-1, \eta, \tau)=f_{0} \delta(\eta-\beta) \text { for all } \tau
$$

and the periodicity constraint 


$$
f(\xi, \eta, 2 \pi) \equiv f(\xi, \eta, 0)
$$

Quantities such as the time-averaged energy distribution $\Gamma(\varepsilon)$ of the ion flux at the cathode could then be obtained from $f(\xi, \eta, \tau)$ by integration.

Condition (38) may be satisfied automatically by expanding $f$ as a Fourier series in time. Regarded as a function of $\tau$, the factor $\alpha H(\xi+\cos \tau)$ in (35) is a "rectangular wave," whose Fourier expansion has coefficients that are polynomials in $\xi$. On substituting these expansions into (35), there results an infinite sequence of coupled equations for the Fourier components $f_{k}(\xi, \eta)$ of the distribution function. Unfortunately, this approach is impractical - the component equations do not have straightforward solutions, and rapid convergence of the partial Fourier sums cannot be expected because of the discontinuous factor in (35).

Nevertheless, we can gain useful insight into the iontransport problem by studying families of trajectories in the $(\xi, \eta)$ "phase plane." Ions are introduced at the point $(\xi, \eta)=(-1, \beta)$ at a uniform rate, independent of the phase $\phi[23]$, and follow the line $\eta=\beta$ initially. The phase-plane trajectories deviate from this line at different locations, determined by the first encounter with the plasma-sheath boundary, and then follow parabolic paths reflecting the acceleration by the sheath field.

The subsequent phase-plane flow consists of alternating families of linear and parabolic segments that "fold" over each other in a complicated manner. Overall, the flow is confined to a bounded region of the $(\xi, \eta)$ plane whose piecewise-smooth boundaries are defined by successive states of ions crossing the plasma-sheath boundary. The phase-plane trajectories are terminated at $\xi=+1$. In the steady state, the disposition of the termination points with respect to the velocity $\eta$ will reflect the incident-ion energy distribution if the initial sampling is uniform in $\phi$.

For given values of $\alpha$ and $\beta$, the phase-plane flow over a full rf cycle may be illustrated by computing a representative set of trajectories, corresponding to a fixed small increment $\Delta \phi$ in phase. An algorithmic procedure for "parsing" these trajectories is described in the Appendix. Figure 2 shows the flows obtained in two typical cases that illustrate the features described above and give rise to the characteristic double-peaked energy distribution (as computed by the simulation program).

On close inspection, Fig. 2 reveals that ion trajectories contributing to the high end of the energy spectrum have one less encounter with the sheath field than those contributing to the low end; the former enjoy a longer uninterrupted final run in the field before impinging on the cathode. We will explore this theme in greater detail below.

Note that although in our model the ion motions have no Hamiltonian description-i.e., the forcing term on the right-hand side of (3) cannot be derived from a potential-their phase-plane flow nevertheless still satisfies Liouville's theorem, since the forcing function has no explicit dependence on the ion velocity [24]. In the present model, the phase-space area occupied by a "packet" of ions injected into the sheath over a small time interval $\Delta t$ is actually degenerate (i.e., one-
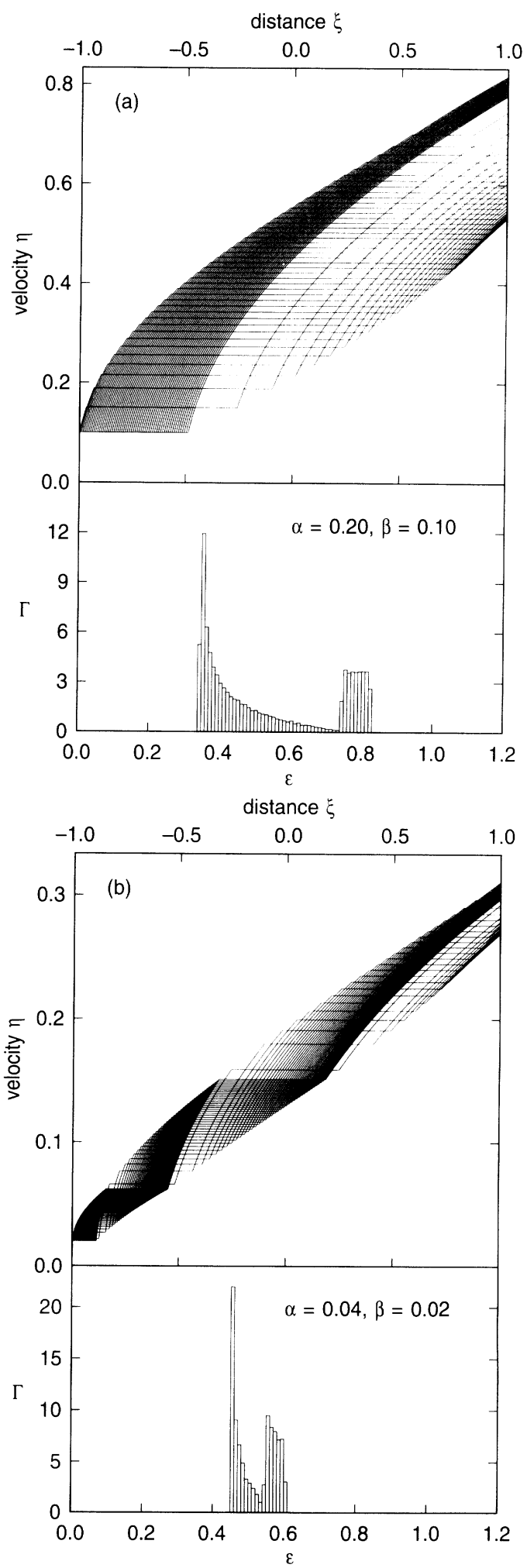

FIG. 2. Portraits of ion trajectories in the $(\xi, \eta)$ phase plane, corresponding to a uniform sampling with respect to the initial phase $\phi$ of the sheath oscillation, for parameter values (a) $\alpha=0.2, \beta=0.1$ and (b) $\alpha=0.04, \beta=0.02$. For clarity, the velocity axis in the latter case has been stretched by a factor 2.5 relative to that of the former. Also shown are corresponding energy distributions of the ion flux generated by the simulation program. 
dimensional) since the ion trajectories depend only on a single initial condition.

\section{B. The initial encounter}

We now give a detailed account of the manner in which ions first cross the plasma-sheath boundary, since this plays an important role in determining their subsequent trajectories. Note that the parameter $\alpha$ does not enter into the problem at this stage.

Let $\xi_{1}(\phi)$ be the function of the initial phase that indicates the location at which an incoming ion first crosses the oscillating plasma-sheath boundary. The corresponding time $\tau_{1}(\phi)$ clearly satisfies

$$
-1+\beta \tau_{1}=-\cos \left(\tau_{1}+\phi\right),
$$

where the left- and right-hand sides represent the location of the ion and of the plasma-sheath boundary, respectively. Since the ion "coasts" during the period $0 \leq \tau<\tau_{1}$, we may substitute $\tau_{1}=\left(1+\xi_{1}\right) / \beta$ in (39) and write

$$
F_{1}\left(\xi_{1}, \phi\right)=\xi_{1}+\cos \left(\frac{1+\xi_{1}}{\beta}+\phi\right)=0
$$

for the equation that determines $\xi_{1}(\phi)$, it being understood that the desired solution is the smallest value of $\xi_{1}$ satisfying (40).

Obviously, the form of the function $\xi_{1}(\phi)$ depends only on the parameter $\beta$. Figure 3 illustrates the variation of this function for a sequence of values in the range $0<\beta \leq 2$, as computed numerically by the procedure described in the Appendix (note that we choose to plot $\xi_{1}$

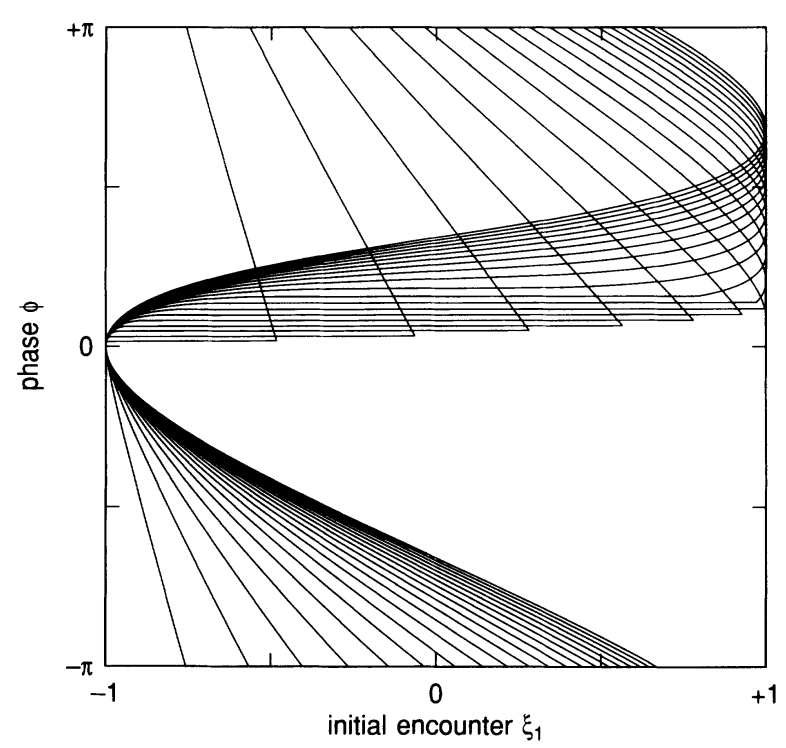

FIG. 3. Location $\xi_{1}$ of the initial encounter of an ion with the oscillating plasma-sheath boundary as a function of the phase angle $\phi$, for values $\beta=0.1,0.2, \ldots, 2.0$ of the initial velocity parameter. To differentiate between the curves, note that $\xi_{1}$ is a decreasing function of $\beta$ at $\phi= \pm \pi$. Observe the discontinuities in $\xi_{1}(\phi)$ at the critical phase $\phi_{\text {crit }}(\beta)$ defined by Eq. (45) when $\beta<1$; for $\beta \geq 1$, there is no jump in $\xi_{1}(\phi)$. against the horizontal axis here, since it represents distance into the sheath).

\section{The critical phase}

Now although $\xi_{1}(\phi)$ has no simple closed-form expression in terms of $\phi$ and the parameter $\beta$, its most important features-evident in Fig. 3-are nevertheless amenable to analysis. In terms of the partial derivatives

$$
\begin{aligned}
& \frac{\partial F_{1}}{\partial \phi}=-\sin \left(\frac{1+\xi_{1}}{\beta}+\phi\right), \\
& \frac{\partial F_{1}}{\partial \xi_{1}}=1-\frac{1}{\beta} \sin \left(\frac{1+\xi_{1}}{\beta}+\phi\right)
\end{aligned}
$$

of (40), the rate of change of $\xi_{1}$ with respect to $\phi$ may be expressed as

$$
\frac{d \xi_{1}}{d \phi}=-\frac{\partial F_{1} / \partial \phi}{\partial F_{1} / \partial \xi_{1}}
$$

As $\phi$ increases from zero, it is seen that this derivative becomes infinite as we approach a certain "critical" phase $\phi_{\text {crit }}(\beta)$, determined by the condition $F_{1}=\partial F_{1} / \partial \xi_{1}=0$. Thus, the critical phase is the solution (if any) to the simultaneous equations

$$
\begin{aligned}
& \cos \left(\frac{1+\xi_{1}}{\beta}+\phi_{\text {crit }}\right)=-\xi_{1}, \\
& \sin \left(\frac{1+\xi_{1}}{\beta}+\phi_{\text {crit }}\right)=\beta .
\end{aligned}
$$

Squaring and adding Eqs. (43) we see that at the critical phase, $\xi_{1}$ must satisfy $\xi_{1}^{2}+\beta^{2}=1$. This has real solutions whenever $\beta \leq 1$, the desired solution being the smaller value

$$
\xi_{1}=-\left(1-\beta^{2}\right)^{1 / 2}
$$

With this value, the right-hand sides of Eqs. (43) are both non-negative, and the argument of the trigonometric terms must lie between 0 and $\pi / 2$. Hence, from the second of Eqs. (43), we may identify this argument as $\sin ^{-1} \beta$, and using (44) we then have

$$
\phi_{\mathrm{crit}}(\beta)=\sin ^{-1} \beta-\frac{1-\left(1-\beta^{2}\right)^{1 / 2}}{\beta}
$$

for the critical phase. Note that $(45)$ is a monotonically increasing function for $0<\beta<1$, having the extreme values

$$
\lim _{\beta \rightarrow 0} \phi_{\text {crit }}(\beta)=0 \text { and } \phi_{\text {crit }}(1)=\frac{\pi}{2}-1 .
$$

When $\beta>1$, there is no critical phase-the derivative $d \xi_{1} / d \phi$ remains finite for all $\phi$ (although it is nevertheless quite large in a certain range of $\phi$ ).

It is interesting to describe the physical significance of the critical phase. Consider at time $\tau=0$ an ion introduced at $\xi(0)=-1$ with speed $\dot{\xi}(0)=\beta$ (where $0<\beta<1$ ), the sheath oscillation being at the critical initial phase 
given by (45). The initial position of the boundary is thus

$$
\begin{aligned}
\xi_{s}(0)= & -\left(1-\beta^{2}\right)^{1 / 2} \cos \left(\frac{1-\left(1-\beta^{2}\right)^{1 / 2}}{\beta}\right) \\
& -\beta \sin \left(\frac{1-\left(1-\beta^{2}\right)^{1 / 2}}{\beta}\right)
\end{aligned}
$$

while its initial speed is

$$
\begin{aligned}
\dot{\xi}_{s}(0)= & \beta \cos \left(\frac{1-\left(1-\beta^{2}\right)^{1 / 2}}{\beta}\right) \\
& -\left(1-\beta^{2}\right)^{1 / 2} \sin \left(\frac{1-\left(1-\beta^{2}\right)^{1 / 2}}{\beta}\right),
\end{aligned}
$$

and it is clear that $-1<\xi_{s}(0)<0$ and $0<\dot{\xi}_{s}(0)<\beta$ when $0<\beta<1$.

Since $\dot{\xi}_{s}(0)<\beta$, the ion initially gains ground on the plasma-sheath boundary, managing to catch up with it at the location $\xi=-\left(1-\beta^{2}\right)^{1 / 2}$. In the meantime, however, the plasma-sheath boundary has been accelerating and has just acquired velocity $\dot{\xi}_{s}=\beta$ at this location. Consequently, the ion has only a glancing encounter with the plasma-sheath boundary at this juncture, being unable to cross it before the latter accelerates ahead.

For phases slightly less than $\phi_{\text {crit }}(\beta)$, ions cross the boundary somewhat before $\xi=-\left(1-\beta^{2}\right)^{1 / 2}$. For phases slightly greater than $\phi_{\text {crit }}(\beta)$, ions "miss" the boundary at this juncture, and only succeed in crossing it much later and thus much nearer the cathode.

We shall denote the jump in $\xi_{1}$ at phase $\phi_{\text {crit }}(\beta)$ by $\Delta \xi_{1}(\beta)$, where it is understood that $0<\beta<1$. Substituting the critical phase (45) for $\phi$ in (40) and setting $\xi_{1}=-\left(1-\beta^{2}\right)^{1 / 2}+\Delta \xi_{1}$, we see that $\Delta \xi_{1}$ is the nonzero (positive) solution to

$\Delta \xi_{1}-\beta \sin \frac{\Delta \xi_{1}}{\beta}+\left(1-\beta^{2}\right)^{1 / 2} \cos \frac{\Delta \xi_{1}}{\beta}=\left(1-\beta^{2}\right)^{1 / 2}$.

It may be verified that $\Delta \xi_{1}(\beta)$ vanishes in the limit $\beta \rightarrow 0$ and when $\beta=1$-otherwise, it has no simple closed-form expression in terms of $\beta$. The behavior of the function $\Delta \xi_{1}(\beta)$ is illustrated in Fig. 4.

We note the existence of a special value $\beta_{*}$ that causes $\xi_{1}$ to attain the value +1 after the jump. This may be identified by setting $\xi_{1}=+1$ in the first of Eqs. (43) and substituting from (45) for the phase. Thus, we find that $\beta_{*}$ is determined by the condition

$$
\beta_{*}=\sin \left(\frac{1+\left(1-\beta_{*}^{2}\right)^{1 / 2}}{\beta_{*}}\right),
$$

and has the approximate numerical value $\beta_{*} \approx 0.724611$. We indicate the location of the value $\beta_{*}$ on the graph of $\Delta \xi_{1}(\beta)$ in Fig. 4 by a dashed line; note that $\Delta \xi_{1}(\beta)$ attains its maximum somewhat before $\beta_{*}$.

\section{The extrema of $\xi_{I}(\phi)$}

From (42) we see that the local extrema of the function $\xi_{1}(\phi)$ are identified by the condition $F_{1}=\partial F_{1} / \partial \phi=0$,

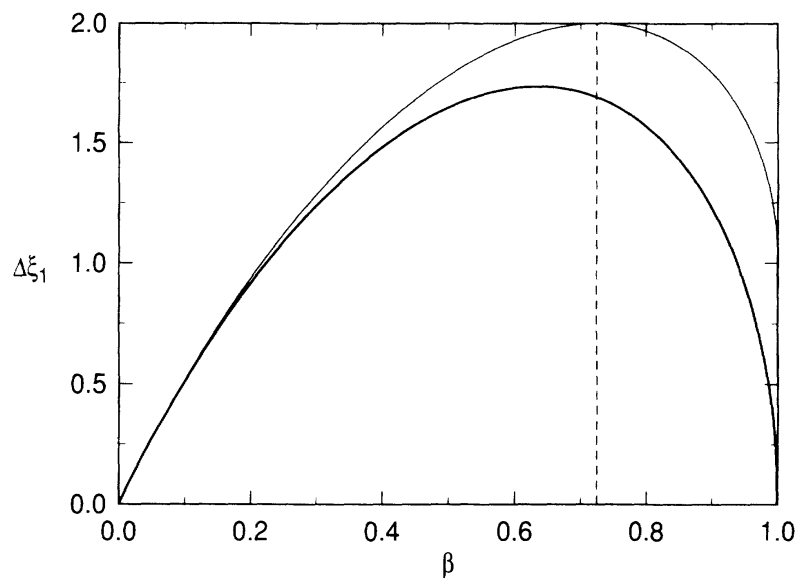

FIG. 4. Variation of the jump $\Delta \xi_{1}(\beta)$ in the location of initial encounters of ions with the oscillating plasma-sheath boundary for phases just below and above the critical phase $\phi_{\text {crit }}(\beta)$ given by (45). The graph represents a numerical solution to (49). Also shown is the quantity $1-\left(1-\beta^{2}\right)^{1 / 2}+\Delta \xi_{1}(\beta)$, which represents the penetration into the sheath of ions introduced at just above the critical phase (thin curve). Thus, an ion injected with velocity $\beta_{*}$ [indicated by the dashed line; see Eq. (50)] at a phase just above $\phi_{\text {crit }}\left(\beta_{*}\right)$ will reach the cathode without crossing the plasma-sheath boundary.

which corresponds to the simultaneous equations

$$
\begin{aligned}
& \cos \left(\frac{1+\xi_{1}}{\beta}+\phi\right)=-\xi_{1}, \\
& \sin \left(\frac{1+\xi_{1}}{\beta}+\phi\right)=0 .
\end{aligned}
$$

From (51) it is clear that the local extrema of $\xi_{1}$ must be either -1 or +1 . A local minimum of $\xi_{1}=-1$ always occurs at the phase

$$
\phi_{\min }(\beta) \equiv 0 \text { for all } \beta>0 \text {. }
$$

Setting $\xi_{1}=+1$ in (51), we see that a local maximum will be achieved when the argument of the trigonometric terms is equal to $+\pi$, i.e., at the phase

$$
\phi_{\max }(\beta)=\pi-\frac{2}{\beta} \text { for } \beta>\beta_{*} .
$$

For $\beta \leq \beta_{*}, \xi_{1}(\phi)$ has no local maximum; its greatest value is that after the "jump" at the critical phase $\phi_{\text {crit }}$, namely $-\left(1-\beta^{2}\right)^{1 / 2}+\Delta \xi_{1}(\beta)$.

Thus, when $\beta>\beta_{*}$, an ion entering at the phase $\phi_{\max }(\beta)$ given by (53) will impinge on the cathode without ever having experienced the sheath field. Corresponding1y, the incident-ion energy distribution $\Gamma(\varepsilon)$ will extend all the way down to $\varepsilon_{\min }$ only when $\beta>\beta_{*}$, regardless of the value of $\alpha$. This explains the low-energy behavior seen in Fig. 1.

\section{Summary of first crossing}

To summarize, the function $\xi_{1}(\phi)$ that gives the location at which an ion injected at phase $\phi$ first crosses the 
oscillating plasma-sheath boundary can be characterized in terms of the parameter $\beta$ as follows.

(i) When $0<\beta \leq \beta_{*}, \xi_{1}(\phi)$ increases monotonically from -1 to $-\left(1-\beta^{2}\right)^{1 / 2}$ as $\phi$ increases from 0 to the critical phase $\phi_{\text {crit }}(\beta)$ given by (45), at which point it jumps suddenly by $\Delta \xi_{1}(\beta)$, an amount defined by Eq. (49). Thereafter, $\xi_{1}(\phi)$ declines monotonically from $-\left(1-\beta^{2}\right)^{1 / 2}+\Delta \xi_{1}(\beta)$ to -1 as $\phi$ further increases from $\phi_{\text {crit }}(\beta)$ through $\pm \pi$ to 0 .

(ii) If $\beta_{*}<\beta \leq 1$, the behavior of $\xi_{1}(\phi)$ for $0 \leq \phi \leq \phi_{\text {crit }}(\beta)$ is qualitatively the same as in the preceding case, i.e., a monotonic increase from -1 to $-\left(1-\beta^{2}\right)^{1 / 2}$ followed by a jump $\Delta \xi_{1}(\beta)$ at the critical phase. After this jump, however, $\xi_{1}(\phi)$ continues to increase, attaining the value +1 at the phase $\phi_{\max }(\beta)$ given by (53). $\xi_{1}(\phi)$ then declines monotonically from +1 to -1 as $\phi$ further increases from $\phi_{\max }(\beta)$ through $\pm \pi$ to 0 .

(iii) When $\beta>1$ there is no critical phase; the graph of $\xi_{1}(\phi)$ is continuous for all $\phi$. Thus, $\xi_{1}$ increases monotonically from -1 to +1 between $\phi=0$ and $\phi=\phi_{\max }(\beta)$, and then declines monotonically from +1 to -1 as $\phi$ further increases from $\phi_{\max }(\beta)$ through $\pm \pi$ to 0 .

\section{Subsequent boundary crossings}

We have characterized the dynamics of initial ion crossings of the oscillating plasma-sheath boundary as a function of the initial phase $\phi$, for any given value of the parameter $\beta$. Although no simple closed-form expression for the location $\xi_{1}$ of the first crossing in terms of $\phi$ and $\beta$ exists, it was nevertheless possible to ascertain the key qualitative aspects of its variation.

\section{Analytical difficulties}

The systematic analysis of subsequent encounters is more difficult, since in general their locations are solutions to concatenated systems of equations in which algebraic and trigonometric expressions are composed in a rather complicated manner. Moreover, both the sheath parameters $\alpha$ and $\beta$ enter into these equations, influencing the existence and nature of their solutions.

Suppose, for example, that at time $\tau_{2}$ and location $\xi_{2}$ an ion injected at phase $\phi$ crosses the boundary for the second time (thus leaving the field). Since the ion is uniformly accelerated during the interval $\left[\tau_{1}, \tau_{2}\right]$ we have

$$
\tau_{2}=\tau_{1}+\frac{\left[2 \alpha\left(\xi_{2}-\xi_{1}\right)+\beta^{2}\right]^{1 / 2}-\beta}{\alpha},
$$

where $\xi_{1}$ is the smallest solution to (40) and $\tau_{1}$ $=\left(1+\xi_{1}\right) / \beta$. The location $\xi_{2}$ of the second encounter is then determined by the condition $\xi_{2}=-\cos \left(\tau_{2}+\phi\right)$, i.e., it is the smallest root greater than $\xi_{1}$ (if any) of the equation

$$
\begin{aligned}
F_{2}\left(\xi_{2}, \phi\right)=\xi_{2}+\cos \left(\frac{\left[2 \alpha\left(\xi_{2}-\xi_{1}\right)+\beta^{2}\right]^{1 / 2}-\beta}{\alpha}\right. \\
\left.+\frac{1+\xi_{1}}{\beta}+\phi\right)=0 .
\end{aligned}
$$

Formally, the dependence of $\xi_{2}$ on $\phi$ and on $\alpha$ and $\beta$ is defined by eliminating $\xi_{1}$ between Eqs. (40) and (55) and then solving the resultant expression for $\xi_{2}$ explicitly in terms of those quantities. Unfortunately, this cannot be accomplished analytically, and the situation grows worse with each successive encounter considered.

However, equations such as (40) and (55) present no special numerical difficulties, and robust algorithmic procedures can be formulated that, given values for $\alpha$ and $\beta$, will solve the entire sequence of equations $F_{1}\left(\xi_{1}, \phi\right)=0$, $F_{2}\left(\xi_{2}, \phi\right)=0, \ldots$, defining each boundary crossing that occurs for the ion trajectory associated with phase $\phi$ (see the Appendix).

Invoking this numerical scheme with small uniform increments in $\phi$ from $-\pi$ to $+\pi$, we can obtain a good impression of how the entire family of ion trajectories corresponding to a full rf cycle will depend on the parameters $\alpha$ and $\beta$-the phase-plane portraits shown in Fig. 2, for example, are obtained in this manner.

\section{Some general observations}

A detailed inspection of the results of many numerical experiments, covering a wide range of the parameters $\alpha$ and $\beta$, leads to the identification of some universal features of the families of ion trajectories that arise in our model.

Henceforth we assume that both $\alpha$ and $\beta$ are appreciably less than unity, since otherwise only an insignificant fraction of the ions (if any) have more than one encounter with the field, and the corresponding ion energy distribution at the cathode is relatively broad and featureless.

Typically, $\alpha$ and $\beta$ must both be less than about $\frac{1}{2}$ for an interesting structure to begin to appear in the family of ion trajectories. Specifically, in this domain of the sheath parameters the following observations hold.

(i) At the critical phase $\phi_{\text {crit }}(\beta)$, the discontinuity in the location $\xi_{1}$ of the initial crossing induces a discontinuity in the locations $\xi_{k}$ of all subsequent crossings $(k=2,3, \ldots)$. Moreover, comparing ions injected at phases slightly above and below $\phi_{\text {crit }}$, the former make their first, second, etc., crossings at locations slightly to the left of those at which the latter make their third, fourth, etc., crossings. In other words,

$$
\lim _{\delta \phi \rightarrow 0} \xi_{k}\left(\phi_{\text {crit }}+\delta \phi\right)=\lim _{\delta \phi \rightarrow 0} \xi_{k+2}\left(\phi_{\text {crit }}-\delta \phi\right)
$$

for $k=1,2, \ldots$ at each $\alpha$ and $\beta$.

(ii) For each $\alpha$ and $\beta$ there exists a second critical phase, $\varphi(\alpha, \beta)$, defined such that all ions introduced at phases in the range $\varphi<\phi<\phi_{\text {crit }}$ will have exactly one more encounter with the field than those outside this range [25]. Comparing ion trajectories at phases slightly above and below $\varphi$, if the latter cross the boundary exactly $n$ times before their final uninterrupted run in the field to the cathode, then the former suffer a further two crossings (i.e., $n+2$ altogether) - losing and regaining the field one additional time before impinging on the cathode. In other words, the final encounter of an ion introduced at phase $\varphi(\alpha, \beta)$ with the oscillating plasma-sheath boundary is a glancing encounter, just as an ion introduced at 
phase $\phi_{\text {crit }}(\beta)$ has a glancing initial encounter with the boundary (as described above).

The above ideas are illustrated by some concrete examples in Fig. 5. Here the vertical axis represents the initial phase $\phi$, while distance $\xi$ into the sheath is measured along the horizontal axis. For each $\phi$, we draw a horizontal line between $\xi_{k}$ and $\xi_{k+1}$ if the ion is "in" the field between those locations (i.e., if $k$ is odd); otherwise, we leave that interval blank. Thus, the total number of encounters with the field, at each $\phi$, is equal to the number of line segments drawn at that phase.

That ions injected at phases between $\varphi(\alpha, \beta)$ and $\phi_{\text {crit }}(\beta)$ lose and regain the field one additional time is manifested by the final protruding "tongue" of white area in the $(\xi, \phi)$ plots of Fig. 5, which touches the line $\xi=+1$. Unfortunately, it is not possible to give a simple closed-form expression for the phase $\varphi(\alpha, \beta)$ that represents the lowermost extent of this tongue.

Consider, for example, the simplest case where ion trajectories can be grouped according to whether they have one or two encounters with the field. As in Eq. (42), the
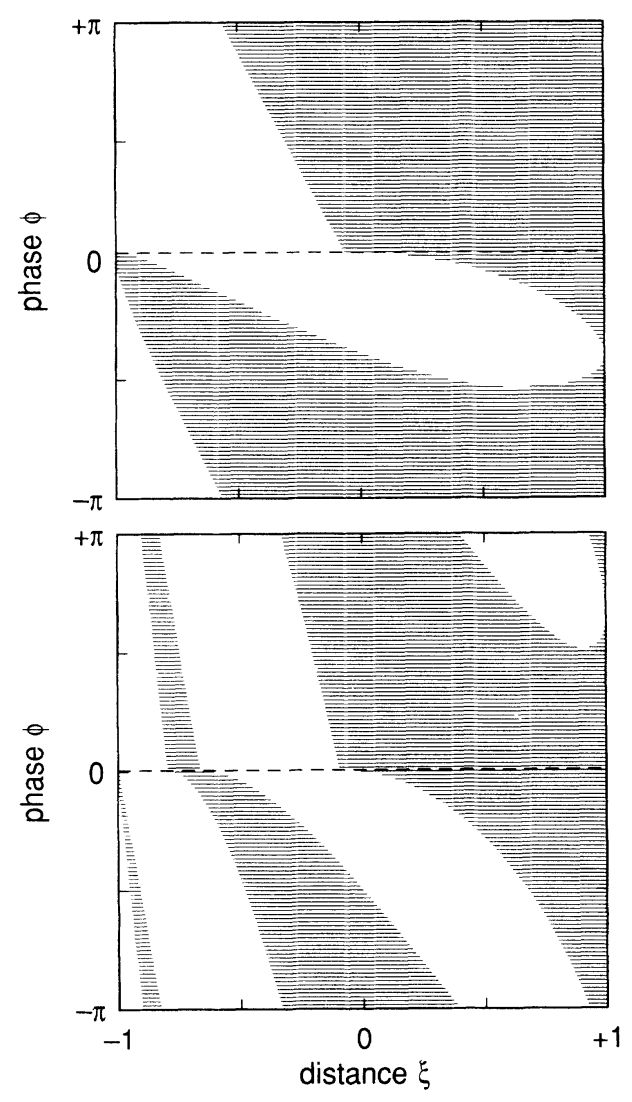

FIG. 5. Disposition of the "coasting" (blank) and "acceleration" (solid) spatial intervals of ion trajectories with respect to the initial phase $\phi$ in two representative cases: $\alpha=\beta=0.2$ (upper), and $\alpha=0.072, \beta=0.036$ (lower). The first critical phase $\phi_{\text {crit }}$ is indicated by a dashed line. In the first case, the second critical phase $\varphi$ is approximately $-\pi / 2$ and the ions have either one or two encounters with field, while in the second case $\varphi \approx+\pi / 2$ and ions have either two or three encounters with the field. derivative $d \xi_{2} / d \phi$ is equal to minus the ratio of the partial derivatives of (55) with respect to $\phi$ and $\xi_{2}$. Identifying the second critical phase $\varphi(\alpha, \beta)$ as the value of $\phi$ at which $\left|d \xi_{2} / d \phi\right| \rightarrow \infty$, we see that $\varphi$ is defined "implicitly" by the system of equations

$\cos \left(\frac{1+\xi_{1}}{\beta}+\varphi\right)=-\xi_{1}$,

$\cos \left(\frac{\left[2 \alpha\left(\xi_{2}-\xi_{1}\right)+\beta^{2}\right]^{1 / 2}-\beta}{\alpha}+\frac{1+\xi_{1}}{\beta}+\varphi\right)=-\xi_{2}$,

$\sin \left(\frac{\left[2 \alpha\left(\xi_{2}-\xi_{1}\right)+\beta^{2}\right]^{1 / 2}-\beta}{\alpha}+\frac{1+\xi_{1}}{\beta}+\varphi\right)$

$$
=\left[2 \alpha\left(\xi_{2}-\xi_{1}\right)+\beta^{2}\right]^{1 / 2},
$$

where the first two members are simply (40) and (55), and the third arises from the condition $\partial F_{2} / \partial \xi_{2}=0$.

Equations (57) are subject to some immediate simplifications - for example, by squaring and summing the last two, we find that, at the second critical phase $\varphi(\alpha, \beta), \xi_{1}$ and $\xi_{2}$ are related by a quadratic equation, and we may make the substitution

$$
\left[2 \alpha\left(\xi_{2}-\xi_{1}\right)+\beta^{2}\right]^{1 / 2}=\left(1-\xi_{2}^{2}\right)^{1 / 2}
$$

for the radical in (57). Nevertheless, it is not possible in general to eliminate $\xi_{1}$ and $\xi_{2}$ between the three Eqs. (57) and thus arrive at a closed-form expression for $\varphi$ in terms of $\alpha$ and $\beta$. This problem can only grow worse as the values of $\alpha$ and $\beta$ are reduced, since the number of encounters grows commensurately and $\varphi(\alpha, \beta)$ is then defined by a larger system of equations.

\section{The role of $\alpha$ and $\beta$}

Fortunately, the qualitative influence of variations in the sheath parameters $\alpha$ and $\beta$ on the family of ion trajectories has a relatively simple description. Namely, reducing $\alpha$ and/or $\beta$ always drives the second critical phase $\varphi(\alpha, \beta)$ to lower values. Thus, a continuous reduction in $\alpha$ and/or $\beta$ will cause $\varphi(\alpha, \beta)$ to cycle from (say) just below $\phi_{\text {crit }}(\beta)$, through $\pm \pi$, back to $\phi_{\text {crit }}(\beta)$, and beyond. Associated with such a cycle, the disposition of the ion trajectories between (say) $N$ and $N+1$ encounters with the field will transmute into one between $N+1$ and $N+2$ encounters as $\varphi(\alpha, \beta)$ passes through $\phi_{\text {crit }}(\beta)$. [Of course, if $\beta$ is varied, the first critical phase $\phi_{\text {crit }}(\beta)$ given by (45) is also changing as $\varphi(\alpha, \beta)$ cycles. But the variation of the former is relatively insignificant compared to that of the latter, being confined to the range (46). When $\beta<<1$, for example, we have $\phi_{\text {crit }}(\beta) \approx \frac{1}{2} \beta+O\left(\beta^{3}\right)$ from (45).]

The cycling of $\varphi(\alpha, \beta)$ is illustrated by representative cases in Figs. 6 and 7, in which one of the parameters $(\alpha, \beta)$ is held fixed while the other is reduced (similar results hold when $\alpha$ and $\beta$ are reduced in unison).

This cycling can also be visualized by plotting $\langle N\rangle$, the phase-averaged number of encounters with the field, against the sheath parameters $\alpha$ and $\beta$. Figure 8 illustrates the behavior of $\langle N\rangle$ against $\log _{10} \beta$, for a logarithmically spaced sequence of 36 fixed $\alpha$ values. The impor- 

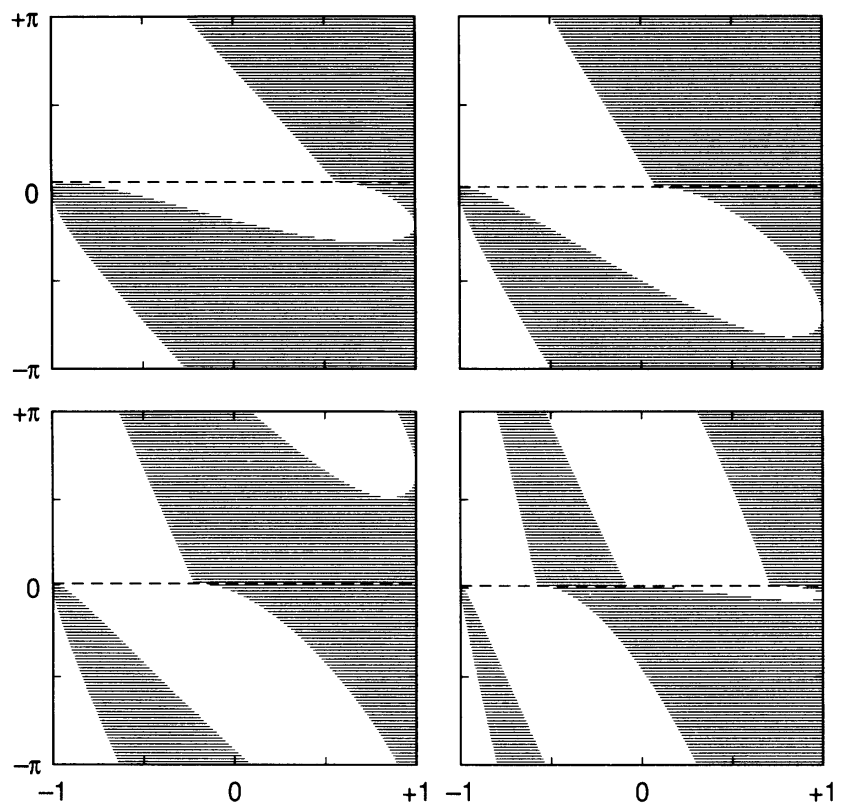

FIG. 6. Illustration of the "cycling" of the second critical phase $\varphi(\alpha, \beta)$ and the accompanying transition of the family of ion trajectories from a division between $N$ and $N+1$ encounters with the field to one between $N+1$ and $N+2$ encounters. Here $\alpha$ is fixed at 0.1 while $\beta$ assumes the values $0.4,0.24,0.16$, and 0.08 (top left, top right, bottom left, and bottom right).

tant point to note is that, associated with each unit increase in $\langle N\rangle$ between consecutive integral values, the second critical phase $\varphi(\alpha, \beta)$ will execute a full cycle. (In Fig. $8,\langle N\rangle$ may be increased by reducing $\beta$ with $\alpha$ fixed - following a single curve to the left, or by reducing $\alpha$ with $\beta$ fixed - moving vertically upward between suc-
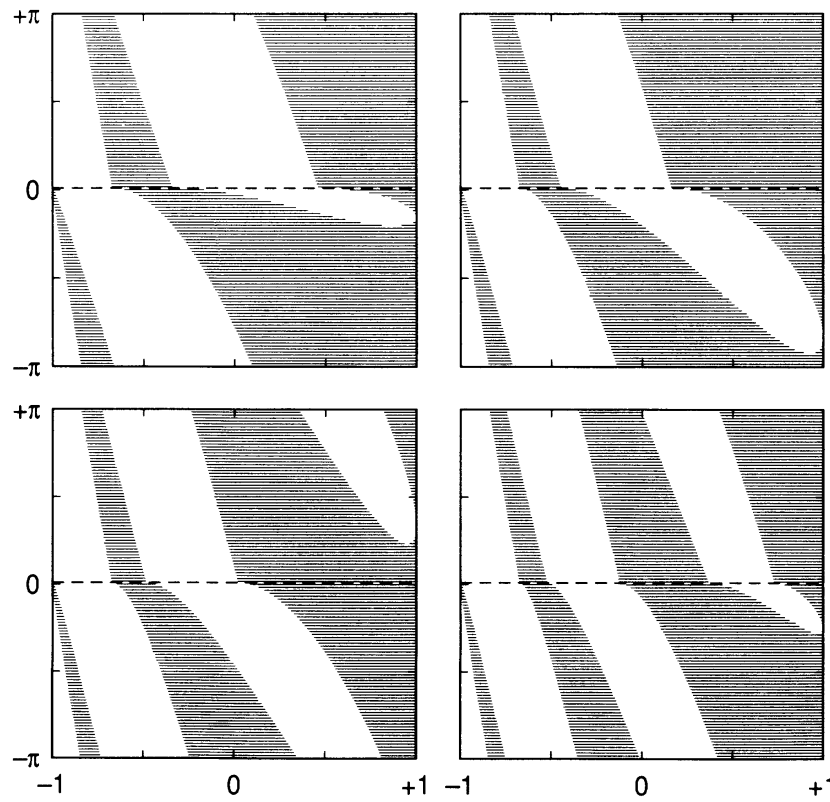

FIG. 7. The same as in Fig. 6 , but with $\beta$ fixed at 0.06 and $\alpha=0.1,0.06,0.042$, and 0.024 (top left, top right, bottom left, and bottom right).

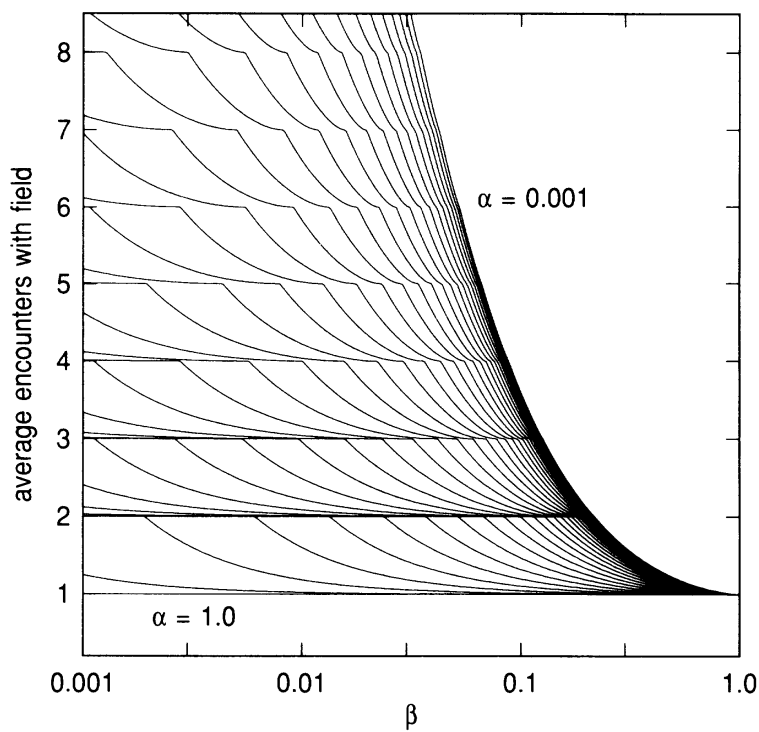

FIG. 8. The dependence of the mean (i.e., phase-averaged) number $\langle N\rangle$ of encounters of ions with the sheath field on the parameters $\alpha$ and $\beta$. The variation of $\langle N\rangle$ with $\beta$ is shown for a sequence of fixed $\alpha$ values. Note that the $\beta$ axis is logarithmic, and the discrete $\alpha$ values for successive curves also correspond to a uniform logarithmic spacing from $\alpha=1.0$ (lowermost curve) to $\alpha=0.001$ (uppermost). Observe that $\langle N\rangle$ increases as we follow an individual curve to the left ( $\beta$ decreasing with $\alpha$ fixed), or as we move vertically upward from one curve to another ( $\alpha$ decreasing with $\beta$ fixed).

cessive curves, or by a combination of such motions.)

In Fig. 9 we plot, in a manner similar to that of Fig. 8, the measured ratio $\Omega(\alpha, \beta)$ of the mean ion-transit time to the rf period - see Eq. (12). By numerical quadrature we find that the high-frequency integral expression (31)

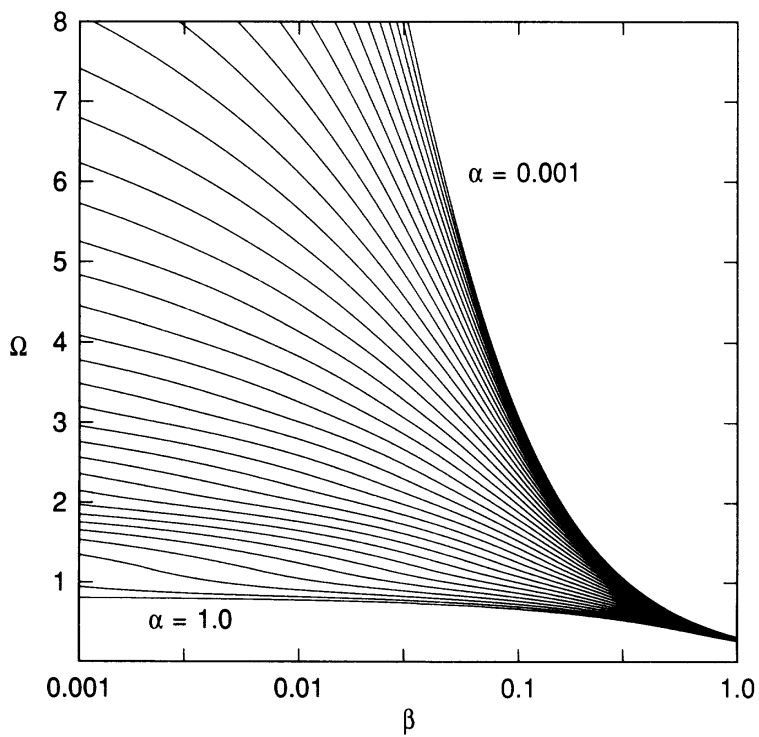

FIG. 9. Variation of the mean-transit-time to rf-period ratio $\Omega$, defined by Eq. (12), with the sheath parameters $\alpha$ and $\beta$. The curves represent a sequence of fixed $\alpha$ values, corresponding to a uniform logarithmic spacing from $\alpha=1.0$ (lowermost curve) to $\alpha=0.001$ (uppermost curve). 
approximates the data of Fig. 9 extremely well over the full range of the sheath parameters shown, i.e., $0.001 \leq \alpha, \beta \leq 1.0$ [the low-frequency form (18) applies to the right of the data shown]. The crude approximation given by (13), however, gives only an order-of-magnitude estimate over much of this range.

Finally, from a practical viewpoint we note from (9) that increasing either the $\mathrm{rf}$ frequency $\omega$ or the mean sheath thickness $d$ causes a diminution of $\alpha$ and $\beta$ (although at different rates). In order to diminish $\alpha$ independently of $\beta$, or vice versa, we need to reduce the mean potential drop $V_{s}$ across the sheath while keeping the Bohm velocity $v_{0}$ (or electron temperature $T_{e}$ ) constant, or vice versa.

\section{INCIDENT-ION ENERGY DISTRIBUTIONS}

Suppose an ion introduced at phase $\phi$ crosses the oscillating plasma-sheath boundary $n$ times, at locations $\xi_{1}<\xi_{2}<\cdots<\xi_{n}$, before impinging on the cathode. Note that $n$ must be odd, since odd and even indices identify crossings where the ion enters and leaves the field, and any ion must be experiencing the field the instant it reaches $\xi=+1$ (if only by convention in the exceptional case that $\xi_{s}=+1$ also at that instant; see the Appendix).

Since the field is spatially constant, the total work done by it on an ion during each acceleration interval is simply proportional to the width $\xi_{k+1}-\xi_{k}$ of that interval ( $k$ odd). Thus, the normalized final energy of an ion injected at phase $\phi$ upon arriving at the cathode is

$\varepsilon(\phi)=\frac{2 \alpha\left[\left(1-\xi_{n}\right)+\cdots+\left(\xi_{4}-\xi_{3}\right)+\left(\xi_{2}-\xi_{1}\right)\right]+\beta^{2}}{4 \alpha+\beta^{2}}$,

where the locations $\xi_{1}, \xi_{2}, \ldots, \xi_{n}$ (and number $n$ ) of the boundary crossings depend on $\phi$ and on the values of the sheath parameters $\alpha$ and $\beta$.

If, as in Sec. II, we assume that $d n_{i} / d \phi$ is a constant (where $d n_{i}$ is the number of ions incident per unit area of the plane $\xi=-1$ between phases $\phi$ and $\phi+d \phi$ in a single rf cycle), then the energy distribution of the ion flux at the cathode is obtained from (59) by

$$
\Gamma(\varepsilon) \propto \sum_{k}\left|\frac{d \varepsilon}{d \phi}\right|_{\phi_{k}}^{-1},
$$

where $\left\{\phi_{k}\right\}$ is the set of finitely many initial phases that yield a prescribed final ion energy $\varepsilon$ at the cathode. It is understood here that $d \varepsilon / d \phi$ on the right-hand side of $(60)$ is to be expressed in terms of $\varepsilon$ - this requires an "inversion" of expression (59). Note that $\Gamma(\varepsilon)$ is singular at any energy $\varepsilon$ that is "stationary" with respect to the initial phase $\phi$, i.e., for which $d \varepsilon / d \phi=0$.

\section{A. The energy-phase relationship}

Although $\varepsilon(\phi)$, as defined by (59), has no explicit representation in terms of elementary functions, we can nevertheless use the numerical results of Sec. III to identify certain generic features of its behavior that help to ex- plain qualitative variations in the shape of $\Gamma(\varepsilon)$ as $\alpha$ and $\beta$ are varied.

Experimentally, there is interest in varying the $\mathrm{rf}$ frequency $\omega$ as a means of controlling the ion energy spectrum. Therefore, we choose here to vary $\alpha$ and $\beta$ simultaneously in such a manner as to represent a uniform increase in $\omega$ with $v_{0}, d$, and $V_{s}$ fixed [see Eq. (9)].

Figure 10 shows representative forms for $\varepsilon(\phi)$ over frequency ranges that induce nearly a full cycle of the second critical phase, $\varphi(\alpha, \beta)$. The following characteristic features of the energy-phase relationship are evident.
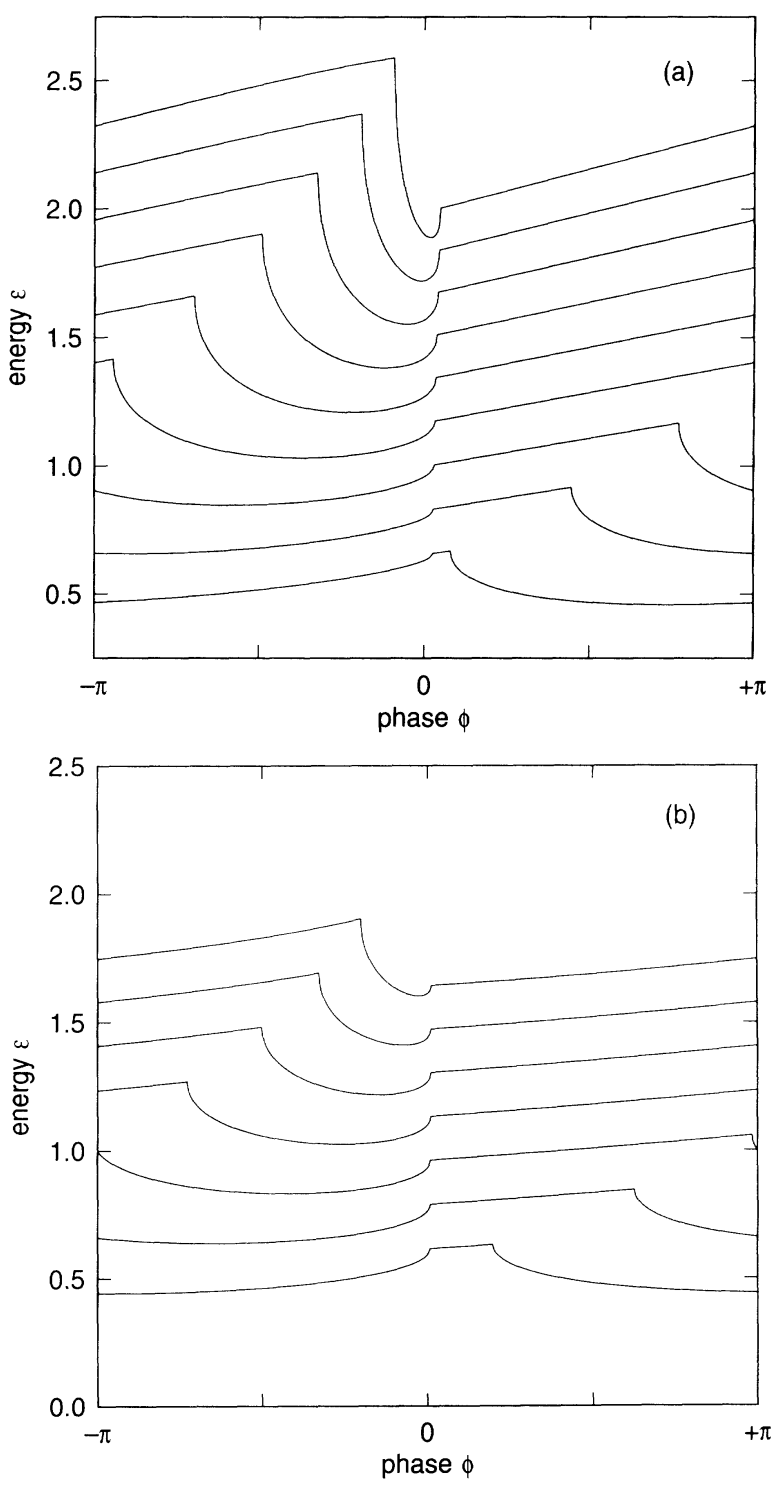

FIG. 10. Variation of the energy-phase relationship with frequency $\omega$ in two representative cases. For clarity, successive graphs are displaced by 0.2 vertical units; the scale on the energy axis holds for the lowermost graph, representing the highest frequency. Case (a) shows a logarithmic progression in eight steps over a full octave in $\omega$, from $(\alpha, \beta)=(0.3,0.3)$ to $(0.075,0.015)$ - successive curves correspond to an increment in $\omega$ of $\sim 9 \%$. Case (b) shows six consecutive $5 \%$ increases in $\omega$, from $(\alpha, \beta)=(0.1,0.06)$ to $\sim(0.056,0.045)$. 
(i) Within the domain $\varphi<\phi<\phi_{\text {crit }}$, corresponding to ions that have $N+1$ encounters with the field, $\varepsilon(\phi)$ has a "parabolic" shape-its second derivative $d^{2} \varepsilon / d \phi^{2}$ is of constant sign but the first derivative $d \varepsilon / d \phi$ changes sign at a clearly defined minimum $\varepsilon_{\text {min }}$.

(ii) Outside the domain $\varphi<\phi<\phi_{\text {crit }}$ (i.e., for ions that have $N$ encounters with the field), $\varepsilon(\phi)$ is monotone and has a nearly uniform variation - the derivative $d \varepsilon / d \phi$ is nearly constant. The range of $\varepsilon(\phi)$ over this domain is between $\varepsilon\left(\phi_{\text {crit }}\right)>\varepsilon_{\min }$ and $\varepsilon(\varphi)=\varepsilon_{\text {max }}$.

This behavior implies that $\varepsilon(\phi)$ shares a noteworthy property with the simple low-frequency relationship (21): there are always two distinct phases $\phi_{1}$ and $\phi_{2}$, dependent on $\varepsilon$, that yield a given energy between $\varepsilon_{\min }$ and $\varepsilon_{\max }$. Thus, the sum $(60)$ for $\Gamma(\varepsilon)$ has terms corresponding to just two phases.

Associated with the local minimum of $\varepsilon(\phi)$, where $d \varepsilon / d \phi=0$, we expect a singularity of the distribution (60) at $\varepsilon_{\min }$ with a "tail" toward higher energies that is more or less prominent according to whether the "parabolic" part of $\varepsilon(\phi)$ is shallow or steep. This is the contribution to $\Gamma(\varepsilon)$ of ions that have $N+1$ encounters to the field. For those that have $N$ encounters, we expect a contribution that is relatively flat (since $d \varepsilon / d \phi \approx$ const) between $\varepsilon\left(\phi_{\text {crit }}\right)\left(>\varepsilon_{\text {min }}\right)$ and $\varepsilon_{\max }=\varepsilon(\varphi)$. [However, it should be noted that when $\varphi(\alpha, \beta)$ passes through $\phi_{\text {crit }}(\beta)$, i.e., during the transition between a division between $N$ and $N+1$ encounters to one between $N+1$ and $N+2$, the behavior of $\varepsilon(\phi)$ is quite complicated and the observations stated above no longer apply in general.]

In case (a) of Fig. 10, the evolution of $\varepsilon(\phi)$ as $\varphi(\alpha, \beta)$ cycles represents a transition from a state in which most ions have one encounter with the field to one in which most ions have two encounters, while in case (b) the transition is between two and three encounters.

\section{B. The energy distribution}

The identification of two "components" to $\Gamma(\varepsilon)$ made above is not entirely clearcut, since it is evident from Fig. 10 that they cover overlapping ranges of energy. Nevertheless, from a qualitative viewpoint it is fair to associate these components with the double-peaked distributions observed experimentally - ions in the high-energy peak having, in general, one less encounter with the field than those in the low-energy peak.

In Fig. 11 we show the energy distributions corresponding to the cases of Fig. 10. In order to truncate the low-energy singularity and provide forms better suited to comparison with experimental data, $(60)$ is represented in histogram form based on the finite sampling interval $\delta \varepsilon=0.005$. The general features of $\Gamma(\varepsilon)$ mentioned above are clearly visible.

Apart from the double-peak structure, no special significance should be attached to the precise shape of the distributions seen in Fig. 11, since they arise from an extremely simple model for the oscillating field. More important is the rapid redistribution of the extent and magnitude of the two "components" to $\Gamma(\varepsilon)$ as $\omega$ is increased and $\varphi$ cycles. Superposed on a systematic overall narrowing, this gives rise to a "quasiperiodic" behavior in the relative prominence of the low- and high-energy peaks when $\alpha$ and $\beta$ are neither comparable to nor much smaller than unity.

Typical physical parameters that characterize the behavior seen in Figs. 10 and 11 are (assuming singly ion-
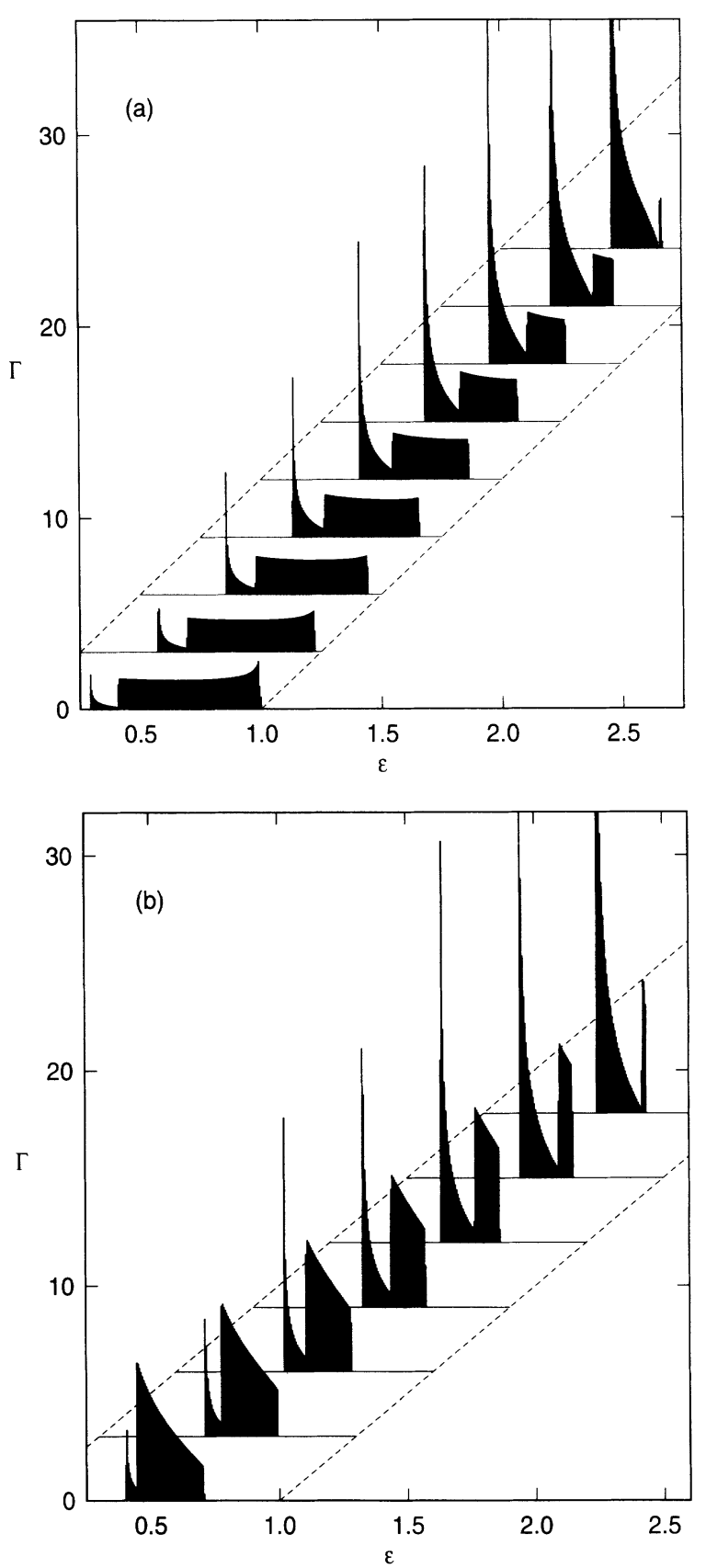

FIG. 11. Normalized energy distributions of the ion flux at the cathode, corresponding to the energy-phase relationships of Fig. 10. For clarity, the distributions at successively higher frequencies are displaced horizontally and vertically, the base line for each plot representing the interval $0 \leq \varepsilon \leq 1$. In case (a) $\omega$ doubles in eight equal steps, while case (b) shows six consecutive increases of $5 \%$ in $\omega$ (see the caption to Fig. 10). 


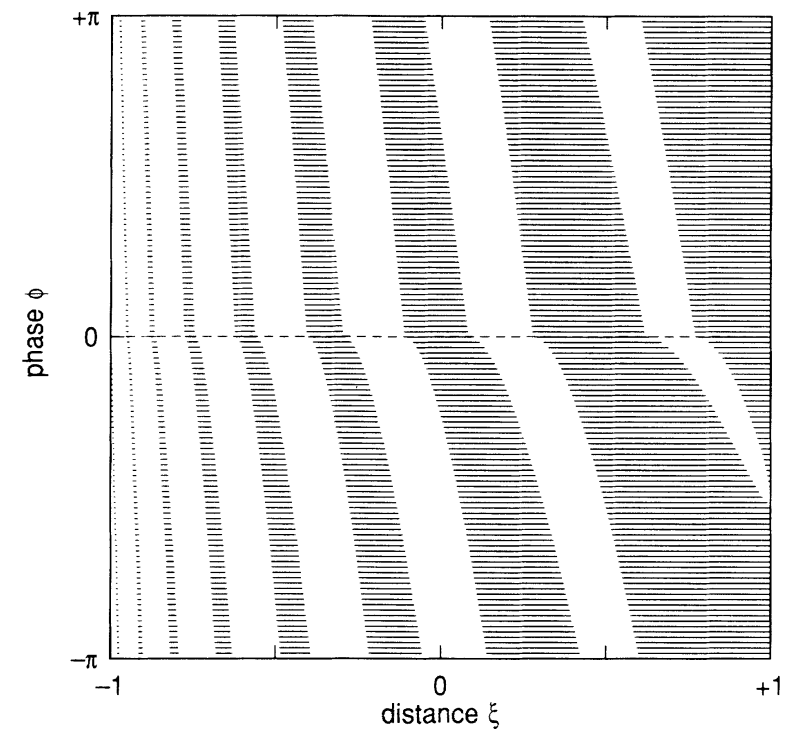

FIG. 12. Distribution of the "coasting" and "acceleration" intervals of ions with respect to phase $\phi$ in a typical highfrequency case, with $\alpha=0.006$ and $\beta=0.008$. Ions have either eight or nine encounters with the field.

ized $\left.\mathrm{O}_{2}\right): V_{s} \approx 60 \mathrm{~V}, d \approx 2.5 \mathrm{~mm}, T_{e} \approx 2 \times 10^{5} \mathrm{~K}$, and $\omega$ increasing from 10 to $20 \mathrm{MHz}$ for case (a); and $V_{s} \approx 270$ $\mathrm{V}, d \approx 3 \mathrm{~mm}, T_{e} \approx 10^{5} \mathrm{~K}$, and $\omega$ increasing from 30 to $\sim 40 \mathrm{MHz}$ for case (b).

Finally, it is interesting to remark on the behavior of our model in the high-frequency regime. Figure 12 shows the distribution with respect to $\phi$ of the coasting and acceleration intervals for the case $\alpha=0.006$ and $\beta=0.008$, in which ions have either 8 or 9 encounters with the field. While such cases are characterized by a significant overall narrowing of the spread in the final ion energies, it is interesting to observe from Fig. 12 that a substantial fraction (half or more) of these energies, proportional to the sum of the acceleration intervals at each $\phi$, are acquired during the last two encounters with the field. Thus, even in the high-frequency regime, the shape of $\varepsilon(\phi)$ is still quite sensitive to the relative positions of the critical phases $\phi_{\text {crit }}$ and $\varphi$, and we may expect the "quasiperiodic" double-peaked structure of $\Gamma(\varepsilon)$ to persist even when the energy spectrum becomes very narrow.

\section{CONCLUDING REMARKS}

Notwithstanding its idealized nature, our rf sheath model incorporates one "realistic" feature that exerts a strong influence on the ion energy spectrum, namely, a relatively sharp plasma-sheath boundary oscillating in sympathy with the $\mathrm{rf}$ excitation. Moreover, through a combination of analysis and numerical experimentation, this model offers an intuitive explanation for the observed qualitative structure of $\Gamma(\varepsilon)$, and some specific predictions concerning its behavior. Recalling the definition of $\alpha$ and $\beta$ in Eq. (9), we now summarize these results.

(i) In the low-frequency limit (defined nominally by $\alpha, \beta>1$ ), the ion energy distribution $\Gamma(\varepsilon)$ admits a sim- ple analytic expression given by (25). The latter is found empirically to be accurate at finite frequencies (see Fig. 1 ), even if $\alpha<1$, provided that $\beta \gtrsim 1$. When $\beta$ decreases below unity, the true distribution differs from (25) primarily in the disappearance of the low-energy spike.

(ii) In the high-frequency limit $(\alpha, \beta<<1)$, we have given here only the trivial distribution (32). A more sophisticated asymptotic analysis [22] of the ion trajectories yields a distribution of the form (33), with a well-defined width and median energy.

(iii) In the "transition" regime, where $\alpha$ and $\beta$ are neither comparable to nor much smaller than unity, $\Gamma(\varepsilon)$ exhibits its most complicated behavior. Two groups of ion trajectories may be identified, delimited by "critical" values of the initial phase $\phi$, that are characterized by $N$ and $N+1$ encounters with the field. The first critical phase $\phi_{\text {crit }}$ depends weakly on $\beta$ only through expression (45). The second critical phase $\varphi$ has no analytic expression in terms of $\alpha$ and $\beta$, but is found empirically to be quite sensitive to both these parameters, cycling continuously as they are reduced (e.g., as $\omega$ is increased)- see Figs. 6, 7, and 8 .

(iv) Qualitatively, ions having $N+1$ encounters with the field contribute a spiked low-energy component to $\Gamma(\varepsilon)$ with a sharp cutoff $\varepsilon_{\text {min }}$ and a tail toward higher energies, while those having $N$ encounters contribute a broader high-energy component. But a continuous redistribution of the relative populations of these two components occurs as $\varphi$ cycles, leading to a "quasiperiodic" behavior in the gross shape of $\Gamma(\varepsilon)$ - superposed on a systematic narrowing (see Fig. 11) - as $\omega$ increases with all other sheath properties held constant.

\section{APPENDIX: PARSING THE ION TRAJECTORIES}

Each ion trajectory across the sheath is characterized by a subdivision of the domain $\xi \in[-1,+1]$ into alternating "coasting" and "acceleration" subintervals. For given values of the parameters $\alpha$ and $\beta$, the number and extent of these subintervals is uniquely determined by the phase angle $\phi$.

The instances $\left\{\tau_{k}\right\}$ at which an ion crosses the oscillating plasma-sheath boundary, and the corresponding ion locations $\left\{\xi_{k}\right\}$ and velocities $\left\{\eta_{k}\right\}$, are determined by the concatenated system of equations

$$
f_{k}(\tau)=\xi_{k}+\eta_{k}\left(\tau-\tau_{k}\right)+\frac{\alpha_{k}}{2}\left(\tau-\tau_{k}\right)^{2}+\cos (\tau+\phi)=0
$$

for $k=0,1, \ldots$, in the dimensionless time $\tau$. The sequence (A1) commences with the initial coefficients $\tau_{0}=0$, $\xi_{0}=-1, \eta_{0}=\beta$, and $\alpha_{0}=0$. Note that the value of $f_{k}(\tau)$ represents the difference between the dimensionless coordinate of the ion and that of the plasma-sheath boundary.

The desired solution to (A1) is defined as the first real root-if any - of $f_{k}(\tau)$ that is greater than $\tau_{k}$. If such a root exists, it corresponds to the value $\tau_{k+1}$. The coefficients $\xi_{k+1}$ and $\eta_{k+1}$ that are required in the succeeding equation $f_{k+1}(\tau)=0$ are then determined by 


$$
\begin{aligned}
& \xi_{k+1}=\xi_{k}+\eta_{k}\left(\tau_{k+1}-\tau_{k}\right)+\frac{\alpha_{k}}{2}\left(\tau_{k+1}-\tau_{k}\right)^{2}, \\
& \eta_{k+1}=\eta_{k}+\alpha_{k}\left(\tau_{k+1}-\tau_{k}\right),
\end{aligned}
$$

while the quantities $\left\{\alpha_{k}\right\}$ in Eqs. (A1)-(A3) are assigned according to

$$
\alpha_{k}=\left\{\begin{array}{l}
0 \text { if } k \text { is even } \\
\alpha \text { if } k \text { is odd } .
\end{array}\right.
$$

Thus, $\left[\xi_{k}, \xi_{k+1}\right]$ represents a coasting or an acceleration interval-and $f_{k}(\tau)$ is correspondingly negative or positive-according to whether $k$ is even or odd. [The case $\phi=0$ is exceptional in that the ion experiences the field immediately; to make it conform to the convention (A4), we assign to it a "null" initial coasting interval by taking $\tau_{1}$ to be zero, rather than the first real root of $f_{0}(\tau)$, and setting $\xi_{1}=-1$ and $\eta_{1}=\beta$.]

The system (A1) terminates when an equation $f_{n}(\tau)=0$ is encountered that has no real solution-or, exceptionally, has a real root $\tau_{n+1}$ that yields $\xi_{n+1}=1$ through Eq. (A2). Note that such a termination can occur only when $n$ is odd, i.e., during an acceleration interval.

In the case that $f_{n}(\tau)$ has no real roots, the ion trajectory is completed (noting that $\alpha_{n}=\alpha$ in such a case) by taking

$$
\tau_{n+1}=\tau_{n}+\frac{\left[2 \alpha\left(1-\xi_{n}\right)+\eta_{n}^{2}\right]^{1 / 2}-\eta_{n}}{\alpha} .
$$

By (A2) and (A3), we then have

$$
\xi_{n+1}=1 \text { and } \eta_{n+1}=\left[2 \alpha\left(1-\xi_{n}\right)+\eta_{n}^{2}\right]^{1 / 2} \text {. }
$$

To solve algorithmically for the first real root of (A1) greater than $\tau_{k}$, or to verify the nonexistence of real roots, we begin by noting that it suffices to consider only the interval $\tau_{k}<\tau \leq \tau_{k}+2 \pi$. This can be seen as follows. Bearing in mind that the polynomial part of (A1) is monotonically increasing with $\tau$, consider the following two cases.

(i) If $\xi_{k}+2 \pi \eta_{k}+2 \pi^{2} \alpha_{k} \leq 1$, the graph of the polynomial part of (A1) increases monotonically from $\xi_{k} \geq-1$ to $\xi_{k}+2 \pi \eta_{k}+2 \pi^{2} \alpha_{k} \leq+1$ as $\tau$ increases from $\tau_{k}$ to $\tau_{k}+2 \pi$, and it is clear that it must intersect the graph of $-\cos (\tau+\phi)$ over the same domain, giving a root of $f_{k}(\tau)$.

(ii) If, otherwise, $\xi_{k}+2 \pi \eta_{k}+2 \pi^{2} \alpha_{k}>1$, it is impossible that $f_{k}(\tau)=0$ for some $\tau>\tau_{k}+2 \pi$, since the magnitude of the polynomial part of (A1) will always exceed that of the trigonometric part for $\tau>\tau_{k}+2 \pi$.

Our strategy for solving (A1) is based on identifying and testing potential isolating intervals for the roots of $f_{k}(\tau)$ on $\tau \in\left(\tau_{k}, \tau_{k}+2 \pi\right)$, as delineated by its extrema on that interval. On differentiating (A1) twice, we have

$$
f_{k}^{\prime \prime}(\tau)=\alpha_{k}-\cos (\tau+\phi)
$$

and we note accordingly that $f_{k}^{\prime \prime}(\tau) \geq 0$ for all $\tau$ if $\alpha_{k} \geq 1 \quad$ [with $f_{k}^{\prime \prime}(\tau)=0$ only at $\tau=2 \pi r-\phi$, where $r=\left\lfloor\left(\tau_{k}+\phi\right) / 2 \pi\right\rfloor+1$, in the case $\left.\alpha_{k}=1\right]$. If $\alpha_{k}<1$, on the other hand, $f_{k}^{\prime \prime}(\tau)$ will always vanish twice on $\tau \in\left(\tau_{k}, \tau_{k}+2 \pi\right]$, namely at

$$
2 \pi p+\cos ^{-1} \alpha_{k}-\phi \text { and } 2 \pi q-\cos ^{-1} \alpha_{k}-\phi,
$$

where $p$ and $q$ are integers chosen such that the values (A8) exceed $\tau_{k}$ but not $\tau_{k}+2 \pi$, and it is understood that $0<\cos ^{-1} \alpha_{k} \leq \pi / 2$. We therefore break down the solution of (A1) into two cases.

Case A: $\alpha_{k} \geq 1$. If $\alpha_{k} \geq 1, f_{k}^{\prime}(\tau)$ is nondecreasing over the open interval $\tau \in\left(\tau_{k}, \tau_{k}+2 \pi\right)$. Thus, $f_{k}^{\prime}(\tau)$ vanishes at most once between $\tau_{k}$ and $\tau_{k}+2 \pi$. We then have

$f_{k}^{\prime}\left(\tau_{k}\right) f_{k}^{\prime}\left(\tau_{k}+2 \pi\right) \geq 0 \Longleftrightarrow f_{k}(\tau)$ is strictly monotone $f_{k}^{\prime}\left(\tau_{k}\right) f_{k}^{\prime}\left(\tau_{k}+2 \pi\right)<0 \Longleftarrow f_{k}(\tau)$ has a single extremum

on $\tau \in\left(\tau_{k}, \tau_{k}+2 \pi\right)$. In the first case, $f_{k}(\tau)$ has either one or no roots between $\tau_{k}$ and $\tau_{k}+2 \pi$ according to whether or not the product $f_{k}\left(\tau_{k}\right) f_{k}\left(\tau_{k}+2 \pi\right)$ is negative; if there is a root it can be approximated to any desired accuracy by the robust bisection procedure [26]. In the second case above, we use bisection to determine the location $\hat{\tau}$ where $f_{k}^{\prime}(\tau)$ vanishes. We then inspect the products

$$
f_{k}\left(\tau_{k}\right) f_{k}(\hat{\tau}) \text { and } f_{k}(\hat{\tau}) f_{k}\left(\tau_{k}+2 \pi\right)
$$

which indicate either one or no roots on the intervals $\left(\tau_{k}, \hat{\tau}\right)$ and $\left(\hat{\tau}, \tau_{k}+2 \pi\right)$, according to whether or not they are negative. As before, roots on these two intervals are determined by bisection (we proceed to the second interval only if no root is indicated on the first).

Case B: $\alpha_{k}<1$. If $\alpha_{k}<1, f_{k}^{\prime \prime}(\tau)$ vanishes at the values (A8). Let $\widetilde{\tau}_{1}$ denote the smaller and $\widetilde{\tau}_{2}$ the greater of those values. Then, according to whether the products

$$
\begin{aligned}
& f_{k}^{\prime}\left(\tau_{k}\right) f_{k}^{\prime}\left(\widetilde{\tau}_{1}\right), \\
& f_{k}^{\prime}\left(\widetilde{\tau}_{1}\right) f_{k}^{\prime}\left(\widetilde{\tau}_{2}\right), \\
& f_{k}^{\prime}\left(\widetilde{\tau}_{2}\right) f_{k}^{\prime}\left(\tau_{k}+2 \pi\right)
\end{aligned}
$$

are negative, $f_{k}^{\prime}(\tau)$ has a root-and $f_{k}(\tau)$ an extremum - on the intervals $\left(\tau_{k}, \widetilde{\tau}_{1}\right),\left(\widetilde{\tau}_{1}, \widetilde{\tau}_{2}\right),\left(\widetilde{\tau}_{2}, \tau_{k}+2 \pi\right)$, respectively. Suppose that $m$ extrema are thus identified $(0 \leq m \leq 3)$, their ordered values being $\hat{\tau}_{1}, \ldots, \hat{\tau}_{m}$ as determined by bisection. Setting $\hat{\tau}_{0}=\tau_{k}$ and $\hat{\tau}_{m+1}=\tau_{k}+2 \pi$, we then have up to four potential isolating intervals $\left(\hat{\tau}_{i}, \hat{\tau}_{i+1}\right), i=0, \ldots, m$, for the roots of $f_{k}(\tau)$. Inspecting the signs of the products

$$
f_{k}\left(\hat{\tau}_{i}\right) f_{k}\left(\hat{\tau}_{i+1}\right) \text { for } i=0, \ldots, m
$$

in order, we can then identify and approximate by bisection the first root - if any - of $f_{k}(\tau)$ on $\left(\tau_{k}, \tau_{k}+2 \pi\right)$.

If no root is found on the open interval $\tau \in\left(\tau_{k}, \tau_{k}+2 \pi\right)$ under case $\mathbf{A}$ or B above, we finally test $\tau_{k}+2 \pi$ as a root by checking to see whether the value of $f_{k}\left(\tau_{k}+2 \pi\right)$ vanishes. If not, we have $k=n$ and the ion trajectory is concluded via Eqs. (A5) and (A6). 
[1] J. W. Coburn and E. Kay, J. Appl. Phys. 43, 4965 (1972).

[2] A. D. Kuypers and H. J. Hopman, J. Appl. Phys. 63, 1894 (1988).

[3] W. M. Holber and J. Forster, J. Vac. Sci. Technol. A 8, 3720 (1990).

[4] VLSI Technology, edited by S. M. Sze (McGraw-Hill, New York, 1988), Chap. 8.

[5] R. T. C. Tsui, Phys. Rev. 168, 107 (1968).

[6] P. Benoit-Cattin and L.-C. Bernard, J. Appl. Phys. 39, 5723 (1968).

[7] A. Metze, D. W. Ernie, and H. J. Oskam, J. Appl. Phys. 65, 993 (1989).

[8] M. J. Kushner, J. Appl. Phys. 58, 4024 (1985).

[9] B. E. Thompson, H. H. Sawin, and D. A. Fisher, J. Appl. Phys. 63, 2241 (1988).

[10] J. I. Ulacia F. and J. P. McVittie, J. Appl. Phys. 65, 1484 (1989).

[11] M. S. Barnes, J. C. Forster, and J. H. Keller, IEEE Trans. Plasma Sci. PS-19, 240 (1991).

[12] If the mean free path for ion-neutral collisions is comparable to or less than the mean sheath thickness, the ion energy distribution exhibits a complex structure with many discrete peaks - see, for example, C. Wild and P. Koidl, Appl. Phys. Lett. 54, 505 (1989); J. Appl. Phys. 69, 2909 (1991); J. Liu, G. L. Huppert, and H. H. Sawin, J. Appl. Phys. 68, 3916 (1990). We shall treat only the simpler collisionless case here.

[13] A. Metze, D. W. Ernie, and H. J. Oskam, J. Appl. Phys. 60, 3081 (1986).

[14] D. Vender and R. W. Boswell, IEEE Trans. Plasma Sci. PS-18, 725 (1990).

[15] M. Surendra and D. B. Graves, IEEE Trans. Plasma Sci. PS-19, 144 (1991).

[16] See, however, M. A. Lieberman, IEEE Trans. Plasma Sci.
PS-16, 638 (1988); PS-17, 338 (1989); C. M. Horwitz and T. Puzzer, J. Vac. Sci. Technol. A 8, 3132 (1990).

[17] D. Bohm, in The Characteristics of Electrical Discharges in Magnetic Fields, edited by A. Guthrie and R. K. Wakerling (McGraw-Hill, New York, 1949), Chap. 3.

[18] C. G. Goedde, A. J. Lichtenberg, and M. A. Lieberman, J. Appl. Phys. 64, 4375 (1988).

[19] C. K. Birdsall and W. B. Bridges, Electron Dynamics of Diode Regions (Academic, New York, 1966), Chap. 1.

[20] In general, if the map $\phi \rightarrow \varepsilon$ is not injective (i.e., a given value of $\varepsilon$ may correspond to more than one value of $\phi$ ), a contribution to the number of ions with energies in the range $\varepsilon$ to $\varepsilon+d \varepsilon$ will be made by each $\phi$ that gives rise to energy $\varepsilon$. In the case of expression (20) there are always two such values, since $\varepsilon(-\phi)=\varepsilon(\phi)$ for each $\phi$, but the derivative $d \varepsilon / d \phi$ differs only in sign at these two values [see Eq. (22)].

[21] R. T. Farouki, S. Hamaguchi, and M. Dalvie, Phys. Rev. A 44, 2664 (1991).

[22] S. Hamaguchi, R. T. Farouki, and M. Dalvie, Phys. Rev. Lett. 68, 44 (1992).

[23] The simultaneous use of the word phase for two different purposes should cause no confusion; it will be clear from the context whether it refers to the ion state $(\xi, \eta)$ or the sheath-oscillation angle $\phi$.

[24] A. J. Lichtenberg, Phase-Space Dynamics of Particles (Wiley, New York, 1969).

[25] If $\varphi(\alpha, \beta)>\phi_{\text {crit }}(\beta)$, it should be understood that by $\varphi(\alpha, \beta)<\phi<\phi_{\text {crit }}(\beta)$ we mean $\varphi(\alpha, \beta)<\phi \leq+\pi$ and $-\pi<\phi<\phi_{\text {crit }}(\beta)$, since $\phi$ is a cyclic variable, confined to the range $-\pi<\phi \leq+\pi$.

[26] G. Dahlquist and $\AA$. Björck, Numerical Methods (Prentice-Hall, Englewood Cliffs, NJ, 1974). 

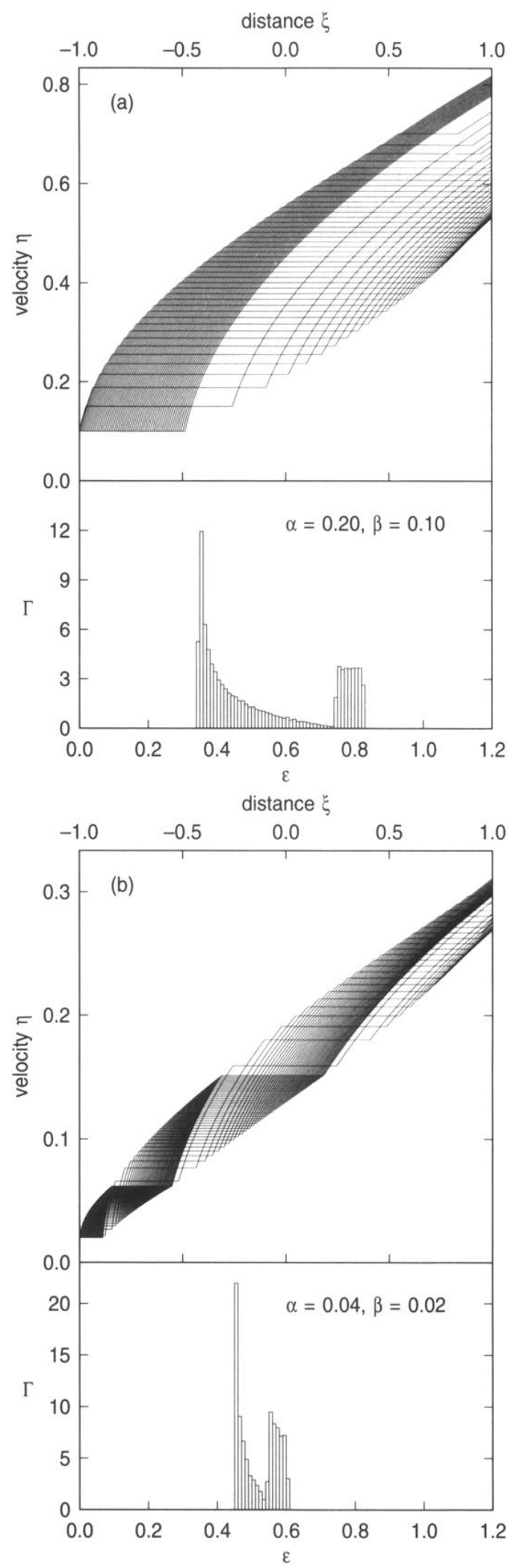

FIG. 2. Portraits of ion trajectories in the $(\xi, \eta)$ phase plane, corresponding to a uniform sampling with respect to the initial phase $\phi$ of the sheath oscillation, for parameter values (a) $\alpha=0.2, \beta=0.1$ and (b) $\alpha=0.04, \beta=0.02$. For clarity, the velocity axis in the latter case has been stretched by a factor 2.5 relative to that of the former. Also shown are corresponding energy distributions of the ion flux generated by the simulation program. 